Additional services for Journal of Fluid Mechanics:

Email alerts: Click here

Subscriptions: Click here

Commercial reprints: Click here

Terms of use : $\underline{\text { Click here }}$

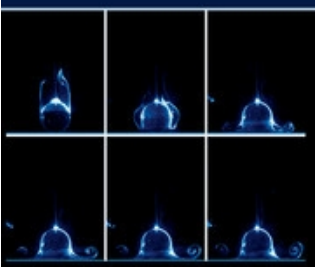

\title{
On vortex/wave interactions. Part 1. Non-symmetrical input and cross-flow in boundary layers
}

\author{
S. N. Brown and F. T. Smith
}

Journal of Fluid Mechanics / Volume 307 / January 1996, pp 101 - 133

DOI: 10.1017/S0022112096000067, Published online: 26 April 2006

Link to this article: http://journals.cambridge.org/abstract S0022112096000067

How to cite this article:

S. N. Brown and F. T. Smith (1996). On vortex/wave interactions. Part 1. Non-symmetrical input and cross-flow in boundary layers. Journal of Fluid Mechanics, 307, pp 101-133 doi:10.1017/ S0022112096000067

Request Permissions : $\underline{\text { Click here }}$ 


\title{
On vortex/wave interactions. Part 1. Non-symmetrical input and cross-flow in boundary layers
}

\author{
By S. N. BROWN AND F. T. SMITH \\ Department of Mathematics, University College, Gower Street, London WC1E 6BT, UK
}

(Received 5 September 1994 and in revised form 11 August 1995)

The paper studies certain effects of non-symmetry on vortex/wave interactions, for inviscid inflexional waves interacting nonlinearly with the vortex component of the mean flow in boundary-layer transition at large Reynolds number. Two types of nonsymmetry are investigated, namely for unequal input wave amplitudes and for small cross-flows. These lead to coupled integro-differential equations for spatial development of the wave amplitudes, which are examined in an essentially equivalent differential form for various degrees of the non-symmetry present. Each type of nonsymmetry can have a significant influence on the nonlinear interaction properties. Special emphasis is given to bounded solutions, and numerous interesting new flow responses are found analytically and computationally. The theory provides a basis for tackling enhanced non-symmetry in the input or stronger cross-flows.

\section{Introduction}

Vortex/wave interactions in boundary layers, channel flows and related motions have attracted considerable attention recently with regard to transitions from laminar flow. These nonlinear interactions arise in various forms, principally with viscousinviscid Tollmien-Schlichting waves or with inviscid inflexional Rayleigh waves as in Hall \& Smith (1988, 1989, 1990, 1991), Walton \& Smith (1992), Blennerhassett \& Smith (1992), Stewart \& Smith (1992), Smith \& Bowles (1992), Walton, Bowles \& Smith (1994), Benney \& Chow (1989), Goldstein \& Choi (1989), Brown et al. (1993), Smith, Brown \& Brown (1993, referred to herein as SBB), Wu (1993a), Wu, Lee \& Cowley (1993) and Khokhlov (1994) in different flow regimes. They all have the common feature, however, that at high Reynolds numbers small-amplitude threedimensional waves are coupled nonlinearly with the mean flow via its unknown longitudinal vortex component. The remarkable smallness of the waves involved, especially in SBB, is in fact one reason on both practical and theoretical grounds for the attention devoted above to vortex/wave interactions, in comparison with the somewhat higher amplitudes connected with nonlinear triple-deck interactions and the still higher amplitudes in Euler-scale interactions. More detailed comparisons are made by Hall \& Smith (1991), Walton \& Smith (1992), SBB and Timoshin \& Smith (1995). A second reason for the theoretical focus on vortex/wave interactions surrounds the qualitative and quantitative links with experiments on transition described by Hall \& Smith (1991), Walton \& Smith (1992), Stewart \& Smith (1992), Smith \& Bowles (1992), for a variety of input conditions. Observations of the significant role of longitudinal vortices in the early stages of some transition paths are given experimentally by Aihara, Tomita \& Ito (1984), Tani \& Sakagami (1962), Klebanoff \& Tidstrom (1959), 
Nishioka, Asai \& Iida (1979) and computationally by Wray \& Hussaini (1984), Spalart \& Yang (1987), Kleiser \& Zang (1991), Sandham \& Kleiser (1992), Rempfer \& Fasel (1994) (and many references therein) for example. Third, and perhaps from a narrower perspective, vortex/wave interactions yield a wide range of interesting new analytical/ computational problems in transitional fluid dynamics.

The concern of this paper is with the effect of non-symmetry on vortex/wave interactions in the presence of inflexional disturbances, as opposed to the symmetric configurations studied by SBB and in the papers referenced in the preceding paragraph. The non-symmetry discussed in the present paper, Part 1, is due either to nonsymmetrical input waves, or to cross-flow in the incident boundary layer. (Part 2, Brown \& Smith 1996, is concerned with the effect of swirl in a jet flow) or both. The effects produced can be substantial in certain parameter regimes. Even quite small cross-flow or swirl for instance is found to have an important influence on the interactions, which is a significant practical point since in reality most incident boundary layers are likely to be three-dimensional to a greater or lesser extent, especially on swept wings, near wing-body junctions or in atmospheric boundary layers (see, for example, Reed \& Saric 1989; Kohama, Saric \& Noos 1991); similar considerations apply to swirling jets and similar flows with their possibility of inducing vortex breakdown. Along with that there is also the need to discover more about the impact of small non-symmetrical disturbances and cross-flow or swirl as well as input frequencies, wavenumbers and disturbance amplitudes, in devices intended to promote or delay transition efficiently.

The theoretical approach used is based on that developed in SBB. A predominantly two-dimensional inflexional boundary layer flowing in the streamwise direction $x$, but with a small amount of cross-flow in the spanwise direction $z$, approaches the neutral station $x=0$ at which small inviscid three-dimensional Rayleigh waves are initiated and interact nonlinearly with the induced three-dimensional mean flow. The waves are of relatively short length and time scales whereas the induced vortex is relatively long and quasi-steady. The small cross-flow and the input non-symmetry are such as to affect the local nonlinear interaction substantially. In due course a study of much stronger cross-flows would be desirable, bringing in full cross-flow modes nonlinearly, cf. Stuart (1963), Hall (1986), Stewart \& Smith (1987), Bassom \& Gajjar (1988), Gajjar (1995) and the strong cross-flows accommodated in Davis \& Smith (1994) for longitudinal vortex interaction with viscous-inviscid waves, but the above approach seems to provide a helpful starting point. The local cross-flow structure then is multizoned in the direction normal to the solid surface or wall $y=0$, as in SBB, with a thin critical layer and two slightly less thin buffer layers lying in the middle of the boundarylayer core of the motion. The resulting nonlinear vortex/wave interaction involves interplay between properties in all the above zones.

The structure of the flow is described in $\$ 2$ below, being as in SBB but with allowance made for non-symmetric wave input and for the small cross-flow. This yields in $\S 3$ a pair of integro-differential complex amplitude equations for the unequal waves present, with or without cross-flow. The equations can be transformed however into two nonlinear second-order ordinary differential equations, as in $\S 4$, limiting forms of which are presented in $\S 5$, guided by the analysis in SBB. Although most of the ultimate solution responses in SBB can still be produced even with non-symmetry active, there is particular interest in the parameter range associated with bounded solutions. Solutions for small cross-flow, governed by a parameter $N$, and near-equal amplitudes, are addressed first in $\S 6$, leading to a system of recurrence relations which are analysed in detail in $\S 7$. These in turn suggest other solutions. Thus, in $\S 8$, more 
general periodic forms are derived; in $\S 9$ forward-marching results are described for moderate values of $L, N$, where the parameter $L$ is the ratio of the real and imaginary parts of a coefficient in the amplitude equations. Further comments are provided in $\S 10$.

\section{The structure of the flow}

The physical background of the problem is exactly as in SBB. The basic equations are the incompressible Navier-Stokes equations in non-dimensional form. With a representative length $L^{*}$ and representative speed $U^{*}$, we write the starred dimensional Cartesian coordinates $\left(x^{*}, y^{*}, z^{*}\right)$, velocity components $\left(u^{*}, v^{*}, w^{*}\right)$, pressure/density ratio $p^{*} / \rho^{*}$ and time $t^{*}$ as

$$
\left.\begin{array}{c}
\left(x^{*}, y^{*}, z^{*}\right)=L^{*}(x, y, z), \quad\left(u^{*}, v^{*}, w^{*}\right)=U^{*}(u, v, w), \\
p^{*} / \rho^{*}=U^{* 2} p, \quad t^{*}=U^{*} t / L^{*} .
\end{array}\right\}
$$

The Reynolds number $R$ defined as

$$
R=U^{*} L^{*} / \nu^{*},
$$

where $\nu^{*}$ is the kinematic viscosity of the fluid, will be taken as large throughout.

As in both Brown et al. (1993) and SBB and the earlier paper of Hall \& Smith (1991), the boundary layer of width $O\left(R^{-\delta}\right)$ develops on a streamwise length scale $O\left(R^{-b}\right)$ where $\delta>\max (0, b)$. The transverse development is on a scale $O\left(R^{-\delta}\right)$ and it is convenient to write

$$
x=R^{-b} \bar{x}, \quad y=R^{-\delta} \bar{y}, \quad z=R^{-\delta} \bar{z} .
$$

A two-dimensional boundary layer, for example a classical boundary layer for which $b=0$ and $\delta=\frac{1}{2}$ in (2.3) or an interactive boundary layer with $b=\frac{3}{8}, \delta=\frac{5}{8}$, is assumed to attain the station $\bar{x}=0$ with a velocity profile $U_{0}(\bar{y})$ that has a point of inflexion at $\bar{y}=\bar{a}_{0}$. This neutrally stable profile initiates the three-dimensional nonlinear development of the flow, a development termed vortex/Rayleigh-wave interaction. Downstream of $\bar{x}=0$ a critical layer is present, consisting of a (unknown a priori) surface $\bar{y}=f(\bar{x}, \bar{z})$ of which the leading edge is the straight line $\bar{x}=0, \bar{y}=\bar{a}_{0}$. Both in SBB and the present work we are concerned with the immediate neighbourhood of the station $\bar{x}=0$ and we define

$$
\bar{x}=\epsilon^{3} x_{1}, \quad \text { where } \quad \epsilon=R^{-(\grave{\delta}-b) / 6},
$$

so that $x_{1}=O(1)$ in what follows. The asymptotic structure of the flow is exactly as in SBB and is illustrated in figure 1 . The core flows have $\bar{y}=O(1)$, while in the buffer layers and critical layer the appropriate scalings are

$$
\bar{y}-f(\bar{x}, \bar{z})=\epsilon^{3 / 2} Y_{1}, \quad \bar{y}-f(\bar{x}, \bar{z})=\epsilon^{2} Y
$$

respectively. There is also a passive viscous layer on the wall of thickness $O\left(\epsilon^{3}\right)$, the effect of which is easily incorporated.

The final important scalings define fast variables in time and in the streamwise direction by

$$
T=R^{3 \grave{\delta}-b-1} t, \quad \alpha_{0} X=R^{\delta-b} \int \alpha(\bar{x}) \mathrm{d} \bar{x}
$$

with the notation $E$ reserved for the exponential

$$
E=\exp \left[\mathrm{i}\left(\alpha_{0} X-\Omega T\right)\right] .
$$

In (2.6) and (2.7), $\Omega$ is a prescribed real frequency and the real wavenumber $\alpha(\bar{x})$ is to be determined as part of the solution. The component of the solution that has a factor 

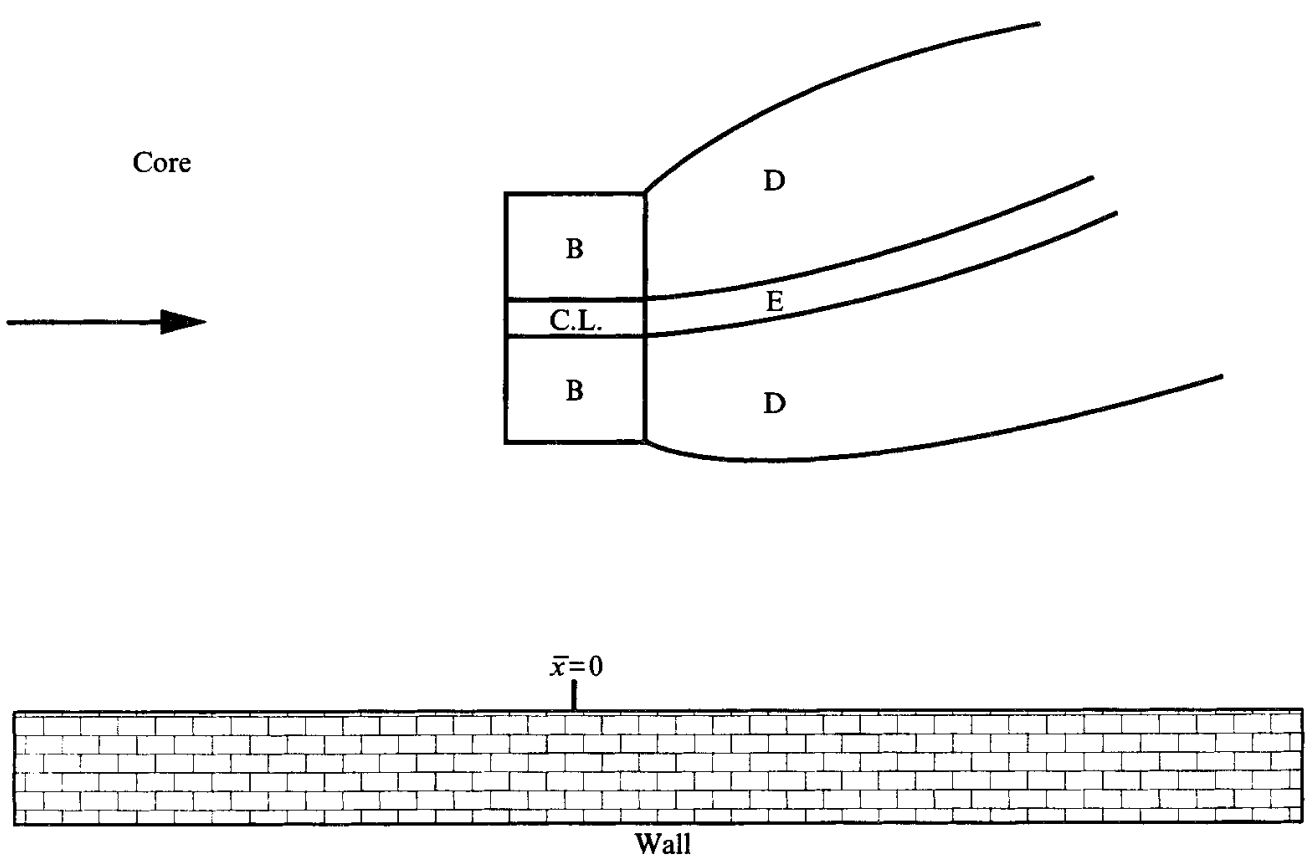

FIGURE 1. Sketch of the short-scale vortex/wave interaction region. The buffer layers B and the critical layer C.L. are in the region $\bar{x}=O\left(\epsilon^{3}\right)$; regions $\mathrm{D}$ and $\mathrm{E}$ represent their continuation into $\bar{x}=O(1)$.

$E$ (or products of its powers and inverses that do not make a zero exponent) is known as the wave, while the $E$-independent component is termed the vortex. In SBB the leading-order wave pressure, i.e. that of the input wave, was taken to be of the form

$$
r\left(x_{1}\right) P_{0}(\bar{y}) E \cos \beta_{0} \bar{z}+\text { c.c. }
$$

where $P_{0}(\bar{y})$ is an eigensolution of Rayleigh's equation and $\beta_{0}$ is a prescribed constant wavenumber in the $\bar{z}$-direction. The result of the investigation was an integrodifferential equation (of cubic nonlinearity) for the amplitude $r\left(x_{1}\right)$. In the present study we replace (2.8) by

$$
\frac{1}{2} P_{0}(y) E\left\{r_{+}\left(x_{1}\right) \mathrm{e}^{\mathrm{i} \beta_{0} \bar{z}}+r_{-}\left(x_{1}\right) \mathrm{e}^{-\mathrm{i} \beta_{0} \bar{z}}\right\}+\text { c.c. }
$$

If we regard (2.8) as typifying a pair of waves at angles $\pm \beta_{0} / \alpha_{0}$ to the mainstream direction with equal amplitude $r\left(x_{1}\right)$, then correspondingly the pair of waves in (2.9) have the possibility of unequal amplitudes $r_{+}\left(x_{1}\right)$ and $r_{-}\left(x_{1}\right)$.

In the following section we describe the amendments that must be made to the integro-differential equation of SBB, firstly due to the input wave (2.9) and secondly due to a small $z$-independent transverse cross-flow component to the oncoming boundary layer.

\section{The integro-differential equation for unequal-amplitude input waves}

For clarity of exposition and ease of verification we describe the adjustments that must be made to the integro-differential equation of SBB in two parts. First, we adopt the input wave (2.9) to replace (2.8), and subsequently incorporate a small, specifically 
$O\left(\epsilon^{3}\right)$, contribution in the $\bar{z}$-direction to the oncoming two-dimensional boundary layer. The reason for the choice of order of magnitude of this contribution is that it affects the core flow at the same level in the series expansion as does the non-parallelism of the basic flow. The corresponding effect on the buffer and critical layer is discussed after (3.13).

The equation obtained and analysed in SBB is

$$
\begin{aligned}
& \frac{\pi b_{3} \gamma_{0}^{2} c_{0}}{\alpha_{0} b_{1}^{2}} r^{\prime}\left(x_{1}\right)+\frac{\left(\frac{2}{3}\right)^{2 / 3} \pi\left(-\frac{2}{3}\right) !}{c_{0}\left(\alpha_{0} b_{1}\right)^{5 / 3}} \beta_{0}^{4}\left(2 \beta_{0}^{2}-\gamma_{0}^{2}\right) r\left(x_{1}\right) \int_{-\infty}^{x_{1}}\left|r^{2}(s)\right| \mathrm{d} s \\
& =b_{1}^{2}\left\{x_{1} r\left(x_{1}\right)\left(G_{a}^{+}-G_{a}^{-}\right)+\mathrm{i} c_{0} r^{\prime}\left(x_{1}\right)\left(G_{b}^{+}-G_{b}^{-}\right)-\frac{P_{0}(0)}{c_{0}^{2}} Q_{w}^{-} r\left(x_{1}\right)\right\},
\end{aligned}
$$

where the constants which comprise the coefficients are as follows:

$$
c_{0}=U_{0}\left(\bar{a}_{0}\right), \quad b_{1}=U_{0}^{\prime}\left(\bar{a}_{0}\right), \quad b_{3}=U_{0}^{\prime \prime \prime}\left(\bar{a}_{0}\right), \quad \gamma_{0}^{2}=\alpha_{0}^{2}+\beta_{0}^{2} .
$$

Also, $G_{a}^{+}-G_{a}^{-}, G_{b}^{+}-G_{b}^{-}$are integrals of the basic flow, and $Q_{w}^{-}$is the contribution from the wall layer. The definitions are

$$
\begin{gathered}
G_{a}^{+}-G_{a}^{-}=2 \int_{0}^{\infty} \frac{P_{0}}{\left(U_{0}-c_{0}\right)^{4}}\left\{\alpha_{0} \alpha_{2}\left(U_{0}-c_{0}\right) P_{0}-\frac{\alpha_{2}}{\alpha_{0}} c_{0} U_{0}^{\prime} P_{0}^{\prime}+\left(U_{1}^{\prime}\left(U_{0}-c_{0}\right)-U_{1} U_{0}^{\prime}\right) P_{0}^{\prime}\right\} \mathrm{d} \bar{y}, \\
G_{b}^{+}-G_{b}^{-}=-\frac{2}{\alpha_{0}} \int_{0}^{\infty} \frac{P_{0}}{\left(U_{0}-c_{0}\right)^{4}}\left\{U_{0}^{\prime} P_{0}^{\prime}-\frac{\alpha_{0}^{2}}{c_{0}}\left(U_{0}-c_{0}\right)^{2} P_{0}\right\} \mathrm{d} \bar{y}
\end{gathered}
$$

and

$$
Q_{w}^{-}=-\gamma_{0}^{2} P_{0}(0)\left(-\mathrm{i} \alpha_{0} c_{0}\right)^{-1 / 2} \text {. }
$$

In (3.3a), $\alpha_{2}$ is the coefficient of $x_{1}$ in the expansion of $\alpha(x)$ in (2.6) as

$$
\alpha(x)=\alpha_{0}+\epsilon^{3} \alpha_{2} x_{1}+o\left(\epsilon^{3}\right)
$$

and $x_{1} U_{1}$ is the $O\left(\epsilon^{3}\right)$ correction to the mainstream in the core region.

In (3.1) the linear terms would arise if there were no vortex-wave interaction. The nonlinear term results from the discontinuity across the critical layer in the transverse shear stress in the buffer region, and its coefficient ( $A$ say) is obtained by solving the equations that are valid in the neighbourhood of the critical layer. In SBB it was necessary to select the coefficient of $\cos \beta_{0} \bar{z}$ in

$$
\left(\frac{\partial^{2} \tilde{p}_{0}}{\partial \bar{z}^{2}}-\alpha_{0}^{2} \tilde{p}_{0}\right) \frac{\partial T_{0}}{\partial \bar{z}}-\frac{\partial \tilde{p}_{0}}{\partial \bar{z}} \frac{\partial^{2} T_{0}}{\partial \bar{z}^{2}}
$$

where

$$
T_{0}\left(x_{1}, \bar{z}\right)=\int_{-\infty}^{x_{1}} J_{0}(s, \bar{z}) \mathrm{d} s
$$

and

$$
J_{0}(s, \bar{z})=\frac{\left(\frac{2}{3}\right)^{2 / 3}\left(-\frac{2}{3}\right) ! 2 \pi}{\left(\alpha_{0} b_{1}\right)^{5 / 3}} \frac{\partial}{\partial \bar{z}}\left(\left|\frac{\partial \tilde{p}_{0}}{\partial \bar{z}}\right|^{2}\right) .
$$

Here

$$
\tilde{p}_{0}\left(x_{1}, \bar{z}\right)=r\left(x_{1}\right) \cos \beta_{0} \bar{z}
$$

and $J_{0}(s, \bar{z})$ is the jump across the critical layer in the transverse shear stress that was mentioned above.

In the new study, in which (2.9) replaces (2.8), to obtain the two equations for $r_{ \pm}\left(x_{1}\right)$ we must now select the coefficients of $\mathrm{e}^{ \pm \mathrm{i} \beta_{0} \bar{z}}$ in (3.6) with

$$
\tilde{p}_{0}\left(x_{1}, \bar{z}\right)=\frac{1}{2}\left\{r_{+}\left(x_{1}\right) \mathrm{e}^{\mathrm{i} \beta_{0} \bar{z}}+r_{-}\left(x_{1}\right) \mathrm{e}^{-\mathrm{i} \beta_{0} \bar{z}}\right\} .
$$


The linear terms in equation (3.1) have $r\left(x_{1}\right)$ replaced by $r_{ \pm}\left(x_{1}\right)$ respectively while the nonlinear terms become, again respectively for the $\mathrm{e}^{ \pm \mathrm{i} \beta_{0} \bar{z}}$ contributions,

$$
r_{\mp}\left(x_{1}\right) \int_{-\infty}^{x_{1}} r_{ \pm}(s) r_{\mp}^{*}(s) \mathrm{d} s,
$$

where the asterisk denotes a complex conjugate.

We now assume that the body is yawed to the oncoming boundary layer so that there is a $\bar{z}$-direction component, $\epsilon^{3} W_{0}(\bar{y})$, for the mainstream velocity. The effect of this modification on the flow in the core is to replace $\alpha_{0} x_{1} U_{1}$ by $\alpha_{0} x_{1} U_{1} \pm \beta_{0} W_{0}$ for the two equations respectively, so that the right-hand side of (3.1) is augmented by a term

where (cf. (3.3))

$$
\pm b_{1}^{2} \beta_{0}\left(G_{c}^{+}-G_{c}^{-}\right) r_{ \pm}\left(x_{1}\right) \text {, }
$$

$$
G_{c}^{+}-G_{c}^{-}=-\frac{2}{\alpha_{0}} \int_{0}^{\infty} \frac{P_{0} P_{0}^{\prime}}{\left(U_{0}-c_{0}\right)^{4}}\left(W_{0}^{\prime}\left(U_{0}-c_{0}\right)-W_{0} U_{0}^{\prime}\right) \mathrm{d} \bar{y} .
$$

In the buffer regions and the critical layer, the only property of $W_{0}$ that is relevant is its value at the critical layer, $W\left(\bar{a}_{0}\right)=g_{0}$ say. In both regions the operator $c_{0} \partial_{x_{1}}$ is replaced by $c_{0} \partial_{x_{1}}+g_{0} \partial_{z}$ which is equivalent to $c_{0} \partial_{x_{1}} \pm \mathrm{i} \beta_{0} g_{0}$ when applied to the terms displayed in (3.10). The first term in (3.1) is a phase-jump contribution to the buffer layer and we must replace $c_{0} r_{ \pm}^{\prime}\left(x_{1}\right)$ here by $c_{0} r_{ \pm}^{\prime}\left(x_{1}\right) \pm \mathrm{i} \beta_{0} g_{0} r_{ \pm}\left(x_{1}\right)$. The integral in the nonlinear term results from the inversion of a Fourier transform with respect to $x_{1}$ obtained as a solution of the buffer-layer equation for the leading contribution to the vortex, namely

$$
\left(c_{0} \partial_{x_{1}}+g_{0} \partial_{\bar{z}}\right) w_{0}=w_{0 Y_{1} Y_{1}} .
$$

Equation (3.14a) is to be solved, as in SBB where $g_{0}=0$, with $w_{0} \rightarrow 0$ as $x_{1} \rightarrow-\infty$ and as $Y_{1}$, the buffer-layer normal coordinate, tends to infinity. Also the jump (3.8) forced by the critical layer leads to a discontinuity $J_{0}\left(x_{1}, \bar{z}\right)$ in $w_{0 Y_{1}}$ across $Y_{1}=0$. Since it follows from (3.8) that $w_{0}$ is a linear combination of terms in $\exp \left( \pm 2 \mathrm{i} \beta_{0} z\right),(3.14 a)$ may easily be solved and (3.11) is now replaced by

$$
r_{\mp}\left(x_{1}\right) \int_{-\infty}^{x_{1}} \exp \left[ \pm \frac{2 \mathrm{i} \beta_{0} g_{0}}{c_{0}}\left(s-x_{1}\right)\right] r_{ \pm}(s) r_{\mp}^{*}(s) \mathrm{d} s
$$

as the nonlinear contribution.

Equation (3.14a) may be recognized as equation (3.7) of Part 2 with $r_{0} \theta$ identified with $\bar{z}$ and $\delta_{0}$ with $g_{0}$. Indeed the analysis of the buffer and critical layers, presented here as a straightforward extension of SBB, may be deduced from the more intricate calculation of Part 2 in the limit $r_{0} \rightarrow \infty$ with $n / r_{0}$ fixed.

Finally we make the transformations

$$
r_{ \pm}\left(x_{1}\right)=t_{ \pm}\left(x_{1}\right) \exp \left[\mp i \frac{\beta_{0} g_{0}}{c_{0}} x_{1}\right]
$$

so that the appropriate analogues of (3.1) are

$$
C t_{ \pm}^{\prime}\left(x_{1}\right)+A t_{\mp}\left(x_{1}\right) \int_{-\infty}^{x_{1}} t_{ \pm}(s) t_{\mp}^{*}(s) \mathrm{d} s+\left(B x_{1}+F_{ \pm}\right) t_{ \pm}\left(x_{1}\right)=0,
$$

which may be compared with (6.1) of SBB. The constants $A, B, C$ have the same values as in SBB where $A$ was real and $C$ complex. In SBB the value of $B$ was taken to be real as there the discussion was restricted to profiles such that the solution in the region 
under consideration had the possibility of a match with the initiation of the Hall-Smith vortex/wave interaction as analysed in BBST. If this restriction is removed and a more general class of downstream development is examined, as it will be here and also was in SBB, then the requirement of a downstream limit with a regular critical layer for a match with the Hall-Smith solution is no longer necessary. The result is that $B$ in (3.16) can be complex. A complex $B$ makes no difference to the analysis of the solutions of the amplitude equation that were carried out in SBB. Also in (3.16)

$$
F_{ \pm}=-\mathrm{i} D \mp b_{1}^{2} \beta_{0}\left\{G_{c}^{+}-G_{c}^{-}+g_{0}\left(G_{b}^{+}-G_{b}^{-}\right)\right\}=-\mathrm{i} D \pm G \text { say. }
$$

Here $D, G$ are real ( $D$ by a change of origin of $x_{1}$ ). In addition $A, B, C, D$ are functions of $\beta_{0}^{2}$ as are $G_{b}^{ \pm}, G_{c}^{ \pm}$, and hence it is only the real parts of $F_{ \pm}$that differ in the constants in the two equations (3.16).

Integro-differential equations resembling (3.16) arise in discussions of the nonlinear evolution of instability modes in laminar boundary layers and shear flows in many of the references cited early in the introduction. The motivation behind the derivation of the corresponding equation of SBB, to which (3.16) reduces in the equal-amplitude case $t_{+}=t_{-}$with $F_{+}=F$, was to analyse the development of the amplitude $t_{+}\left(x_{1}\right)$ on a streamwise length scale that permitted a match downstream with the small- $x$ solution of the Hall-Smith vortex/wave-interaction equations which hold when $x$ of (2.3) is $O(1)$. In the Hall-Smith structure it is anticipated that the coupled reaction between the oblique Rayleigh waves and the developing mean-flow results in an evolving regular viscous critical layer of equilibrium type, and that the self-sustaining interaction will persist to large distances downstream. In SBB, of which the present study is a generalization to unequal amplitudes of the input waves and to cross-flow, the shorter lengthscale $x=O\left(\epsilon^{3}\right)$, i.e. $x_{1}=O(1)$, is considered, and critical-layer interaction, again of a viscous equilibrium type, between two oblique waves of amplitude $O\left(\epsilon^{7}\right)$ forces a spanwise-dependent (vortex) contribution of smaller order $O\left(\epsilon^{8}\right)$. In addition to the Hall-Smith limit the equation of SBB generates three other possible solution paths of interest in their own right.

By contrast the integro-differential equation of Wu et al. (1993) involves multiple integrations, a more complicated kernel, but no non-parallel term. These authors study oblique input waves of equal amplitude in an analogous problem of an unsteady shear layer but work on a shorter length scale (there a time scale) equivalent to $x=O\left(\epsilon^{4}\right)$ here. In their situation the critical layer is of non-equilibrium type, since the streamwise gradients are larger, the buffer layer is absorbed into the critical layer, and the spanwise-dependent mean flow is induced at the same order, $O\left(\epsilon^{6}\right)$, as the input wave. The inviscid limit of these authors' amplitude equation reduces to that of Goldstein \& Choi (1989), and in a discussion of the relation between their approach and the Hall-Smith vortex/wave interaction theory, they demonstrate that in a certain viscous limit it can be reconciled with that of SBB without the non-parallel term. Further explanation of the various length (time) scales involved is given by $\mathrm{Wu} \&$ Cowley (1995). The same shorter $x$-scale and non-equilibrium critical layer is involved in the resonant-triad studies of, for example, Wu (1995).

Most studies to date have been confined to symmetrical situations, i.e. the oblique waves make equal angles with the direction of the undisturbed mainstream and are of equal amplitude. An example without these restrictions is that of Wu (1993b) which, although an examination of the development of a triad of Tollmien-Schlichting waves rather than Rayleigh waves as considered here, leads to a pair of equations of the form (3.16) together with one for the two-dimensional wave. His equations effectively have $C$ purely imaginary, and his numerical solutions show that interaction with the two- 
dimensional wave can result in two oblique waves of unequal input amplitude evolving to an equal-amplitude state. Equations (3.16) have complex constants and a nonparallel term and hence a very rich solution space. For any non-zero cross-flow, i.e. non-zero $G$ in (3.17), equal amplitudes will not be a possibility here.

The following sections are devoted to a study of equations (3.16) with emphasis on parameter values that will result in solutions that are expected to persist at large distances downstream, and on the effects of the cross-flow.

\section{The governing ordinary differential equations}

In this section we rewrite equations (3.16) as differential equations for the modulus and phase of $t_{ \pm}$by eliminating the integrals and separating the resultant equations into their real and imaginary parts. We define successively

$$
\begin{gathered}
t_{ \pm}\left(x_{1}\right)=\rho_{ \pm}\left(x_{1}\right) \exp \left[\mathrm{i} \theta_{ \pm}\left(x_{1}\right)\right], \quad R_{ \pm}=\rho_{ \pm}^{2}, \\
S=\rho_{+}^{2}+\rho_{-}^{2}, \quad T=\rho_{+}^{2}-\rho_{-}^{2}
\end{gathered}
$$

and for the constants appearing in (3.16) we let $C=\lambda+\mathrm{i} \mu, B=\sigma+\mathrm{i} \tau$ and define $L, M$, $N$ by

$$
L^{2}=\lambda^{2} / \mu^{2}, \quad M=(\lambda \sigma+\mu \tau) /\left(\lambda^{2}+\mu^{2}\right), \quad N=G \lambda /\left(\lambda^{2}+\mu^{2}\right) .
$$

The equations for the phases $\theta_{ \pm}$are then

$$
\left.\begin{array}{c}
4 \lambda \mu R_{+} \theta_{+}^{\prime}=\lambda^{2} T^{\prime}-\mu^{2} S^{\prime}+2(D \mu+G \lambda) S+2 x_{1}(\lambda \sigma T-\mu \tau S), \\
4 \lambda \mu R_{-} \theta_{-}^{\prime}=-\lambda^{2} T^{\prime}-\mu^{2} S^{\prime}+2(D \mu-G \lambda) S-2 x_{1}(\lambda \sigma T+\mu \tau S) .
\end{array}\right\}
$$

The change of origin

$$
(\lambda \sigma+\mu \tau) x_{1}-D \mu=(\lambda \sigma+\mu \tau) x
$$

enables the constant $D$ to be eliminated from the modulus equations. With a final transformation

these equations are

$$
S(x)=S_{1}(x) \mathrm{e}^{-M x^{2}}, \quad T(x)=T_{1}(x) \mathrm{e}^{-M x^{2}}
$$

$$
\begin{gathered}
\left(S_{1}^{2}-T_{1}^{2}\right) S_{1}^{\prime \prime}=S_{1}\left(S_{1}^{\prime 2}+L^{2} T_{1}^{\prime 2}\right)+4 N S_{1}\left\{L^{2} S_{1} T_{1}^{\prime}+T_{1} S_{1}^{\prime}+N\left(1+L^{2}\right) S_{1}^{2}\right\} \\
-A \lambda \mathrm{e}^{-M x^{2}}\left(S_{1}^{2}-T_{1}^{2}\right)^{2} /\left(\lambda^{2}+\mu^{2}\right) \\
\left(S_{1}^{2}-T_{1}^{2}\right) T_{1}^{\prime \prime}=-T_{1}\left(T_{1}^{\prime 2}+L^{-2} S_{1}^{\prime 2}\right)+2 N\left\{\left(1-L^{-2}\right) T_{1}^{2} S_{1}^{\prime}\right. \\
\left.-\left(1+L^{-2}\right) S_{1}^{2} S_{1}^{\prime}-2 S_{1} T_{1} T_{1}^{\prime}-2 N\left(1+L^{-2}\right) S_{1}^{2} T_{1}\right\}
\end{gathered}
$$

with the parameters $L, M, N, A \lambda /\left(\lambda^{2}+\mu^{2}\right)$ remaining.

In SBB, all the solutions of equations (4.7) were found in the special case of $N=0$ with also $T_{1}=0$. This is the completely symmetric situation with zero cross-flow, and two waves of equal amplitude equally inclined to the mainstream direction. With $N=$ $T=0$ the behaviour of the solutions depended only on the signs of $M$ and $A \lambda$. With $M>0$ and $A \lambda<0$ there were four possibilities. The first, in which the solution terminates at a saddle point in the phase plane as $x \rightarrow \infty$ with a non-zero constant limit for $S$ (not for $S_{1}$ ), is that which matches with the initiation solution of the Hall-Smith (1991) wave/vortex equations as discussed in Brown et al. (1993). This solution is unique. The other solutions for $S$ either decay both as $x \rightarrow \pm \infty$, exist between two algebraic singularities, or decay at one end and terminate at a finite value of $x$ at the other. With $M>0$, but $A \lambda>0$, only the decaying solutions are possible. If $M<0$ and $A \lambda<0$ all solutions for $S$ have two terminating algebraic singularities but if $A \lambda>0$ all solutions for $S$ have the interesting property of being periodic. These periodic solutions 
were the subject of extensive discussion in SBB as it was believed that the resulting selfsustaining waves on a flow which is nevertheless changing structure would be applicable to the early phases of laminar-turbulent transition.

Equations (4.7) may be regarded as replacing equations (3.16). In addition, however, because of the differentiation of (3.16) involved above, there are constraints on the appropriate starting conditions for (4.7); these correspond to requiring $C t_{+}^{\prime}=$ $-\left(B x_{1}+F_{ \pm}\right) t_{ \pm}$at the start of the interaction where nonlinear effects are negligible. It may be shown that this reduces to

$$
S_{1}^{\prime}+2 N T_{1}=0, \quad T_{1}^{\prime}+2 N S_{1}=0
$$

as a starting requirement. Further reference to (4.8) is made in the following section.

In the following section we submit the governing equations (4.7) to a further simplification with a view to computing and analysing solutions of a periodic or selfperpetuating form.

\section{A limiting form of the governing equations}

The solutions of equations (4.7) discussed in SBB that persisted on a large streamwise scale were (with $N=T=0$ ) the periodic solutions for $S$. These, at large values of the typical amplitude, exhibited long regions of predominantly vortex flow interrupted by rapid vortex/wave interactions which continually moderated the vortex flow. The first of these is a non-parallel phenomenon while the second is a short-scale quasiparallel readjustment. For this interpretation of the solution it was necessary first to consider $x \gg 1$, and then to have $M<0$ and $A \lambda>0$ so that the periodicity conditions were satisfied. We now discuss equations (4.7) under similar conditions. We assume that $x$ remains within an $O(1)$ (or less) distance of $X_{0}$ where $X_{0} \gg 1$, change the origin to $X_{0}$ and replace the factor $\mathrm{e}^{-M x^{2}}$ multiplying the quartic terms in (4.7) by $\mathrm{e}^{-M X_{0}^{2}}$. Because of the scaling properties of (4.7) it is then sufficient to replace $A \lambda \mathrm{e}^{-M X_{0}^{2}} /\left(\lambda^{2}+\mu^{2}\right)$ by \pm 1 and, by analogy with SBB since this produced the solutions of greatest interest, we choose the positive sign. Although this argument can be carried through regardless of the sign of $M$, the resulting solutions are expected to be of most significance when $M<0$ because if $M>0$ it is likely, (see (4.6)), that $S, T$ will be exponentially small. Negative $M$ was required for the periodic solutions of SBB.

With $A \lambda \mathrm{e}^{-M x^{2}} /\left(\lambda^{2}+\mu^{2}\right)$ replaced by unity, as explained above, (4.7) can, by addition and subtraction, be put in the form

$$
\begin{aligned}
& \tilde{A} \widetilde{B} \tilde{A}^{\prime \prime}=\frac{1}{8}\left[L^{2}\left(\tilde{A}^{\prime}-\widetilde{B}^{\prime}\right)^{2}+\left(\tilde{A}^{\prime}+\widetilde{B}^{\prime}\right)^{2}\right]\left[\left(1-L^{-2}\right) \tilde{A}+\left(1+L^{-2}\right) \tilde{B}\right]-\widetilde{A}^{2} \widetilde{B}^{2} \\
& +\frac{1}{2} N\left\{\left(\tilde{A^{\prime}}-\tilde{B}^{\prime}\right)\left(L^{2}(\tilde{A}+\widetilde{B})^{2}-\tilde{A}^{2}+\tilde{B}^{2}\right)\right. \\
& \left.+\left(\tilde{A^{\prime}}+\widetilde{B}^{\prime}\right)\left(\widetilde{A^{2}}-\widetilde{B}^{2}-2 \tilde{A} \widetilde{B}-L^{-2}\left(\tilde{A}^{2}+\widetilde{B}^{2}\right)\right)\right\} \\
& +\frac{1}{2} N^{2}(\tilde{A}+\widetilde{B})^{2}\left\{\left(1+L^{2}\right)(\tilde{A}+\tilde{B})-\left(1+L^{-2}\right)(\tilde{A}-\tilde{B})\right\}, \\
& \tilde{A} \tilde{B} \tilde{B}^{\prime \prime}=\frac{1}{8}\left[L^{2}\left(\tilde{A^{\prime}}-\tilde{B}^{\prime}\right)^{2}+\left(\tilde{A}^{\prime}+\tilde{B}^{\prime}\right)^{2}\right]\left[\left(1+L^{-2}\right) \tilde{A}+\left(1-L^{-2}\right) \tilde{B}\right]-\tilde{A}^{2} \tilde{B}^{2} \\
& +\frac{1}{2} N\left\{\left(\tilde{A}^{\prime}-\widetilde{B}^{\prime}\right)\left(L^{2}(\tilde{A}+\widetilde{B})^{2}+\tilde{A}^{2}-\widetilde{B}^{2}\right)\right. \\
& \left.+\left(\tilde{A}^{\prime}+\widetilde{B}^{\prime}\right)\left(\tilde{A}^{2}-\tilde{B}^{2}+2 \tilde{A} \widetilde{B}+L^{-2}\left(\tilde{A}^{2}+\widetilde{B}^{2}\right)\right)\right\} \\
& +\frac{1}{2} N^{2}(\tilde{A}+\tilde{B})^{2}\left\{\left(1+L^{2}\right)(\tilde{A}+\tilde{B})+\left(1+L^{-2}\right)(\tilde{A}-\tilde{B})\right\}
\end{aligned}
$$

containing the two parameters $L, N$, where

$$
\tilde{A}=S_{1}+T_{1}, \quad \widetilde{B}=S_{1}-T_{1} .
$$

It must be remembered throughout that both $\tilde{A}$ and $\tilde{B}$ are non-negative since they are defined as positive multiples of $\rho_{+}^{2}$ and $\rho_{-}^{2}$ in (4.2). Also, although the system (5.1), 
(5.2) is associated with extreme conditions such as large typical amplitudes, input far upstream, large-amplitude input, and/or extreme values of $A, M$ in (4.7), these conditions are in fact very interesting physically, particularly the applications to increased amplitudes. This point is made in SBB. As there, and as indicated in the previous paragraph, we would expect a non-parallel-flow effect to re-enter, from (4.7), even further downstream probably on the longer $O\left(X_{0}\right)$ scale in $x$. Finally, the starting requirements (4.8) are not expected to impose any great restrictions on the current solution. Some of the solutions of (5.1) that we shall obtain satisfy them automatically in the region under consideration (see for example figure $3(a, c)$ for which $N=0$ and $S_{1}^{\prime}$ and $T_{1}^{\prime}$ repeatedly have common zeros). Those solutions that do not satisfy (4.8) in the present region of interest are assumed to correspond to wave profiles that have evolved in an upstream region where the requirements were satisfied.

Although equations (5.1) appear complicated it is possible to gain from them some feeling, by inspection, of the effect of the cross-flow parameter $N$. With $N=0$, there is, for all $L$, a non-trivial solution with $\tilde{A}=\tilde{B}$ and non-constant. This is the solution of SBB with $T_{1}=0$. When cross-flow is present $\tilde{A}$ and $\widetilde{B}$ are not interchangeable unless the sign of $N$, or alternatively that of $x$, is also changed. If we recall that $\widetilde{A}, \widetilde{B}$ represent amplitudes of imposed waves that are equally inclined to the $x$-axis, whereas the presence of the cross-flow implies a bias towards the direction of one or other of these waves, then the skew-symmetry is not unexpected. We note here, and it will be of interest in $\S 9$ below, the existence of the simple symmetric solution $\tilde{A}=\widetilde{B}=$ $4\left(1+L^{2}\right) N^{2}$.

In the following section we demonstrate analytically the existence of periodic solutions of (5.1) by asymptotic methods in which the asymptotically small parameter chose, $\tilde{\epsilon}$ say, is a measure of the distance between the functions $\tilde{A}$ and $\widetilde{B}$. It will, in addition, for the consistency of the analysis, be necessary to assume that $N=O(\tilde{E})$. The resulting period is asymptotically large.

\section{Solutions for small cross-flow with amplitudes $\tilde{A}$ and $\tilde{B}$ approximately equal}

When $\tilde{A}$ and $\tilde{B}$ are taken to be equal and the cross-flow $N$ set equal to zero, equations (5.1) have the solution

$$
\tilde{A}=\tilde{B}=a \operatorname{sech}^{2} \frac{a^{1 / 2}}{\sqrt{ } 2}(x-b),
$$

where $a, b$ are arbitrary constants. This solution can, by suitable interpretation of the constants $a, b$, be recognized as that obtained in $\S 7$ of SBB when the periodic solutions of large amplitude were examined for $x \gg 1$. It corresponds to a region of rapid vortex/wave interaction and is followed in that study by a long region of non-parallel predominantly vortex flow with negligible wave action. The cycle is then repeated because in the situation considered there (which has $T_{1}=N=0$ in (4.7)) all solutions of (5.1) with $A \lambda>0$ are periodic functions of $x$. Our aim in this section is to show that the symmetric solution (6.1) can be used to construct non-symmetric solutions of (5.1) that are periodic functions of $x$. We shall find that there are also solutions of (5.1) that are not periodic functions.

The structure of the solutions of (5.1) that have $\tilde{A} \approx \tilde{B}$ is as follows. There are humps in which the sech ${ }^{2}$ solution of (6.1) is appropriate separated by troughs in which $\tilde{A}$ and $\widetilde{B}$ both oscillate. The solution in each hump must be matched to those in the adjoining troughs. Equations (5.1) have the property that if $\tilde{G}(x)$ is a solution so is $a \tilde{G}\left(a^{1 / 2} x\right)$ for 
any constant $a$, so for simplicity we shall take $a=1$ in (6.1) so that the maximum height in a hump is unity. It emerges that the matching requires the same value of $a$ for each hump.

\section{(a) The solutions in the humps}

We let the successive humps have their maxima at $x=0, s_{1}, s_{2}, \ldots$, with, for the $n$th hump

$$
\begin{aligned}
\tilde{A}\left(s_{n}\right)+\tilde{B}\left(s_{n}\right)=2, & \tilde{A}^{\prime}\left(s_{n}\right)+\tilde{B}^{\prime}\left(s_{n}\right)=0, \\
\tilde{A}\left(s_{n}\right)-\tilde{B}\left(s_{n}\right)=\gamma_{n} \tilde{\epsilon}, & \tilde{A}^{\prime}\left(s_{n}\right)-\tilde{B}^{\prime}\left(s_{n}\right)=\delta_{n} \tilde{\epsilon},
\end{aligned}
$$

where $\tilde{\epsilon}$ acts as a book-keeping parameter measuring the distance between $\tilde{A}$ and $\tilde{B}$. We assume $0<\tilde{\epsilon} \ll 1$. At present $\gamma_{n}$ and $\delta_{n}$ are at our disposal.

The solution in the $n$th hump is thus

$$
\tilde{A}(x)+\tilde{B}(x)=2 \operatorname{sech}^{2} \frac{1}{\sqrt{2}}\left(x-s_{n}\right)
$$

from (6.1) with $a=1$, and from (5.1) on linearization

where

$$
\tilde{A}(x)-\tilde{B}(x)=\tilde{\epsilon} \tilde{C}(x),
$$

$$
\tilde{C}^{\prime \prime}(x)+\frac{2}{L} \tanh ^{2} \frac{1}{\sqrt{ } 2}\left(x-s_{n}\right) \tilde{C}(x)=\tilde{N} \operatorname{sech}^{2} \frac{1}{\sqrt{ } 2}\left(x-s_{n}\right) \tanh \frac{1}{\sqrt{ } 2}\left(x-s_{n}\right)
$$

if we set

$$
N=2^{-5 / 2} \tilde{\epsilon} \tilde{N} /\left(1+L^{-2}\right)
$$

The boundary conditions on (6.5) are that

$$
\tilde{C}\left(s_{n}\right)=\gamma_{n}, \quad \tilde{C}^{\prime}\left(s_{n}\right)=\delta_{n}
$$

to satisfy $(6.2 b)$. The solution of (6.5) may be written down by variation of parameters in terms of the solutions of the homogeneous equation. We denote these by $\phi_{0}(x), \phi_{1}(x)$ where $\phi_{0}\left(s_{n}\right)=1, \phi_{0}^{\prime}\left(s_{n}\right)=0$, and $\phi_{1}\left(s_{n}\right)=0, \phi_{1}^{\prime}\left(s_{n}\right)=1$. To match with the solutions in the troughs we shall require the asymptotic forms of $\phi_{0}(x), \phi_{1}(x)$ when $\left|x-s_{n}\right| \gg 1$, which are

$$
\phi_{0}(x) \approx q \sin \left(\frac{2}{L}\left(\frac{x-s_{n}}{\sqrt{ } 2}-y_{0}\right)\right), \quad \phi_{1}(x) \approx r \sin \left(\frac{2}{L}\left(\frac{x-s_{n}}{\sqrt{ } 2}-y_{1}\right)\right),
$$

where $y_{0}, y_{1}, q, r$ are constants depending on the value of $L$ and are easily found numerically. It is not difficult to ascertain their asymptotic forms when $L \gg 1$ and $L \ll 1$ and this is undertaken in the Appendix.

The solution of (6.5) that satisfies (6.7) is

$$
\tilde{C}(x)=-\phi_{0}(x) \int_{s_{n}}^{x} H \phi_{1} \mathrm{~d} x_{1}+\phi_{1}(x) \int_{s_{n}}^{x} H \phi_{0} \mathrm{~d} x_{1}+\gamma_{n} \phi_{0}(x)+\delta_{n} \phi_{1}(x),
$$

where we have written $H$ for the right-hand side of (6.5). It follows from (6.8) that for $x-s_{n} \gg 1$

$$
\tilde{C}(x) \approx\left(\gamma_{n}-I_{1}\right) q \sin \frac{2}{L}\left(\frac{x-s_{n}}{\sqrt{2}}-y_{0}\right)+\left(\delta_{n}+I_{0}\right) r \sin \frac{2}{L}\left(\frac{x-s_{n}}{\sqrt{2}}-y_{1}\right)
$$

but for $x-s_{n} \ll-1$,

Here

$$
\tilde{C}(x) \approx\left(\gamma_{n}+I_{1}\right) q \sin \frac{2}{L}\left(-\frac{x-s_{n}}{\sqrt{2}}-y_{0}\right)-\left(\delta_{n}+I_{0}\right) r \sin \frac{2}{L}\left(-\frac{x-s_{n}}{\sqrt{ }}-y_{1}\right) .
$$

$$
I_{0,1}=\int_{s_{n}}^{\infty} H \phi_{0,1} \mathrm{~d} x_{1}
$$


and the facts that $\phi_{0}$ is an even function of $x-s_{n}$ whereas $\phi_{1}, H$ are odd, have been used. The expressions (6.10), (6.11) will be required for the match to the solutions in the troughs on each side of the hump centred on $x=s_{n}$.

(b) The solution in the troughs

To leading order the solution in the troughs is obtained by ignoring the cross-flow terms proportional to $N$ in (5.1) and the nonlinear terms $\widetilde{A}^{2} \widetilde{B}^{2}$ on the grounds that they are smaller by a factor $\tilde{\epsilon}$. The general solution of the resulting equations is, in the $n$th trough,

$$
\begin{gathered}
\tilde{A}+\tilde{B}=\mu_{n} \cosh \left(\lambda_{n}\left(x-t_{n}\right)\right), \\
\tilde{A}-\tilde{B}=\mu_{n} \sin \left(\frac{\lambda_{n}}{L}\left(x-t_{n}\right)+h_{n}\right)
\end{gathered}
$$

with four arbitrary constants $\lambda_{n}, \mu_{n}, t_{n}, h_{n}$. We now match the solutions (6.13), (6.14) to those in the hump, with suffix $n$ say, behind this $n$th trough, and to those in the hump in front of it with suffix $(n+1)$.

Matching $\tilde{A}+\widetilde{B}$ of (6.13) as $x-t_{n} \rightarrow-\infty$ with the solution (6.3) as $x-s_{n} \rightarrow \infty$ first shows that $\lambda_{n}=\sqrt{ } 2$ and then gives a relation between $\mu_{n}$, the minimum value of $\tilde{A}+\widetilde{B}$ in the $n$th trough, and the difference between the $x$-coordinates of this minimum and the top of the previous hump. A similar relation is obtained by matching with (6.3), with $n+1$ replacing $n$, as $x-t_{n} \rightarrow \infty$ and $x-s_{n+1} \rightarrow-\infty, s_{n+1}$ now being the position of the top of the succeeding hump. The two relations are

$$
\mu_{n} / 16=\exp \left[-\sqrt{ } 2\left(t_{n}-s_{n}\right)\right]=\exp \left[-\sqrt{ } 2\left(s_{n+1}-t_{n}\right)\right]
$$

From (6.15) we first deduce that the trough is symmetrically between the two humps, and that the distance between the lowest point of the trough and the highest point of the hump is large, since (6.13) was derived on the assumption that $\mu_{n}=O(\tilde{\epsilon})$.

Further relations are found by the match of $\tilde{A}-\tilde{B}$. As the $n$th hump is exited on the right, i.e. as $x-s_{n} \rightarrow \infty$, it follows that the asymptotic form of $(\tilde{A}-\tilde{B}) / \tilde{\epsilon}$ is exactly as given by the expression for $\widetilde{C}(x)$ in (6.10). However, as the $(n+1)$ th hump is exited on the left, i.e. as $x-s_{n} \rightarrow-\infty$, the asymptotic form of $(\tilde{A}-\widetilde{B}) / \tilde{\epsilon}$ is given by the expression in (6.11) with $(n+1)$ for $n$. Both these asymptotic forms must match with $\widetilde{A}-\tilde{B}$ in (6.14). The match of the amplitudes leads to

$$
\begin{aligned}
\frac{\mu_{n}}{\tilde{\epsilon}} & =\left[\left(\gamma_{n}-I_{1}\right)^{2} q^{2}+\left(\delta_{n}+I_{0}\right)^{2} r^{2}+2\left(\gamma_{n}-I_{1}\right)\left(\delta_{n}+I_{0}\right) q r \cos \frac{2}{L}\left(y_{0}-y_{1}\right)\right]^{1 / 2} \\
& =\left[\left(\gamma_{n+1}+I_{1}\right)^{2} q^{2}+\left(\delta_{n+1}+I_{0}\right)^{2} r^{2}-2\left(\gamma_{n+1}+I_{1}\right)\left(\delta_{n+1}+I_{0}\right) q r \cos \frac{2}{L}\left(y_{0}-y_{1}\right)\right]^{1 / 2} .
\end{aligned}
$$

Because of the simple sinusoidal form of $\tilde{A}-\tilde{B}$ in the trough (see (6.14)) the phases of the asymptotic forms as the humps are exited must also be identical. The result of the identification is that

$$
\Gamma_{n}^{+}+\Gamma_{n+1}^{-}+(2 m+1) \pi=\frac{\sqrt{ } 2}{L}\left(s_{n+1}-s_{n}\right)
$$

where $\Gamma_{n}^{+}, \Gamma_{n+1}^{-}$are defined by

$$
\begin{gathered}
\frac{\mu_{n}}{\tilde{\epsilon}} \mathrm{e}^{\mathrm{i} \Gamma_{n}^{+}}=\left(\gamma_{n}-I_{1}\right) q \mathrm{e}^{2 \mathrm{i} y_{0} / L}+\left(\delta_{n}+I_{0}\right) r \mathrm{e}^{2 \mathrm{i} y_{1} / L}, \\
\frac{\mu_{n}}{\tilde{\epsilon}} \mathrm{e}^{\mathrm{i} \Gamma_{n+1}^{-}}=\left(\gamma_{n+1}+I_{1}\right) q \mathrm{e}^{2 \mathrm{i} y_{0} / L}-\left(\delta_{n+1}+I_{0}\right) r \mathrm{e}^{2 \mathrm{i} y_{1} / L},
\end{gathered}
$$

and $m$ is any integer as long as it is large. 
The interpretation of $(6.15)-(6.18)$ is as follows. If the conditions (6.2) are given at the hump $x=s_{n}$, i.e. $\tilde{\varepsilon} \gamma_{n}, \tilde{\varepsilon} \delta_{n}$ are given, then the position of the next hump, $x=s_{n+1}$ and the corresponding values $\gamma_{n+1}, \delta_{n+1}$ may be obtained in terms of them. First, note that, since $\mu_{n}$ is known in terms of $\gamma_{n}, \delta_{n}$ from (6.16), (6.15) leads to

$$
s_{n+1}-s_{n}=\sqrt{ } 2 \log \left(16 / \mu_{n}\right) \text {. }
$$

If we then write (6.17) as an equation for $\Gamma_{n+1}^{-}$, in terms of $\Gamma_{n}^{+}$which is known from (6.18a), and successively calculate $\sin \Gamma_{n+1}^{-}, \cos \Gamma_{n+1}^{-}$, equation (6.18b) may be solved as a pair of linear equations for $\gamma_{n+1}, \delta_{n+1}$. The procedure may be repeated from hump to hump. In the following section we examine solutions of this system of recurrence relations, showing that it has both periodic and non-periodic solutions. Subsequently we use the periodic solutions to construct periodic solutions of the system (5.1) of differential equations.

To complete the solution in the trough we require the constant $h_{n}$ in (6.14). Since the sinusoidal form of $\widetilde{A}-\widetilde{B}$ persists from the $n$th to the $(n+1)$ th hump right through the $n$th trough it is only necessary to match the phase of $\widetilde{A}-\widetilde{B}$ to that given by $\widetilde{C}(x)$ in (6.10) as $x-s_{n} \rightarrow \infty$ on leaving the $n$th hump. The result of the match is that

$$
h_{n}=\frac{\sqrt{ } 2}{L}\left(t_{n}-s_{n}\right)-\Gamma_{n}^{+}
$$

Here $t_{n}-s_{n}$ is given by (6.15) and the match with the succeeding hump follows automatically on use of (6.17).

\section{The recurrence relations}

The system (6.18) is simpler in appearance if we write $\tilde{\epsilon} q \gamma_{n}=\gamma, \tilde{\epsilon} q \gamma_{n+1}=\tilde{\gamma}$, $\tilde{\epsilon} r\left(\delta_{n}+I_{0}\right)=\delta, \tilde{\epsilon} r\left(\delta_{n+1}+I_{0}\right)=\tilde{\delta}, \mu_{n}=\mu, \tilde{\epsilon} q I_{1}=I$ so that, upon solving $(6.18 b)$ for $\tilde{\gamma}, \tilde{\delta}$ as suggested at the end of the preceding section, we obtain

$$
\begin{aligned}
\tilde{\gamma}+I & =\left\{(\gamma-I) \sin \frac{2}{L}\left(y_{0}+y_{1}-s\right)+\delta \sin \frac{2}{L}\left(2 y_{1}-s\right)\right\} / \sin \frac{2}{L}\left(y_{0}-y_{1}\right), \\
\tilde{\delta} & =\left\{(\gamma-I) \sin \frac{2}{L}\left(2 y_{0}-s\right)+\delta \sin \frac{2}{L}\left(y_{0}+y_{1}-s\right)\right\} / \sin \frac{2}{L}\left(y_{0}-y_{1}\right) .
\end{aligned}
$$

Here

$$
s=\log (16 / \mu)
$$

and, from (6.16),

$$
\begin{aligned}
\mu^{2} & =(\gamma-I)^{2}+\delta^{2}+2(\gamma-I) \delta \cos \frac{2}{L}\left(y_{0}-y_{1}\right), \\
& =(\tilde{\gamma}+I)^{2}+\tilde{\delta}^{2}-2(\tilde{\gamma}+I) \tilde{\delta} \cos \frac{2}{L}\left(y_{0}-y_{1}\right) .
\end{aligned}
$$

Given (7.3a) then $(7.3 b)$ is not independent of (7.1).

It is now clear that, given $\gamma, \delta$ we may calculate $\mu$ from $(7.3 a), s$ from (7.2) and then $\tilde{\gamma}, \delta$ from (7.1). Thus conditions at the $(n+1)$ th hump are obtained from those at the $n$ th. To deal with the $(n+2)$ th hump we update $\gamma, \delta$ to $\tilde{\gamma}, \tilde{\delta}$ and repeat the cycle. 

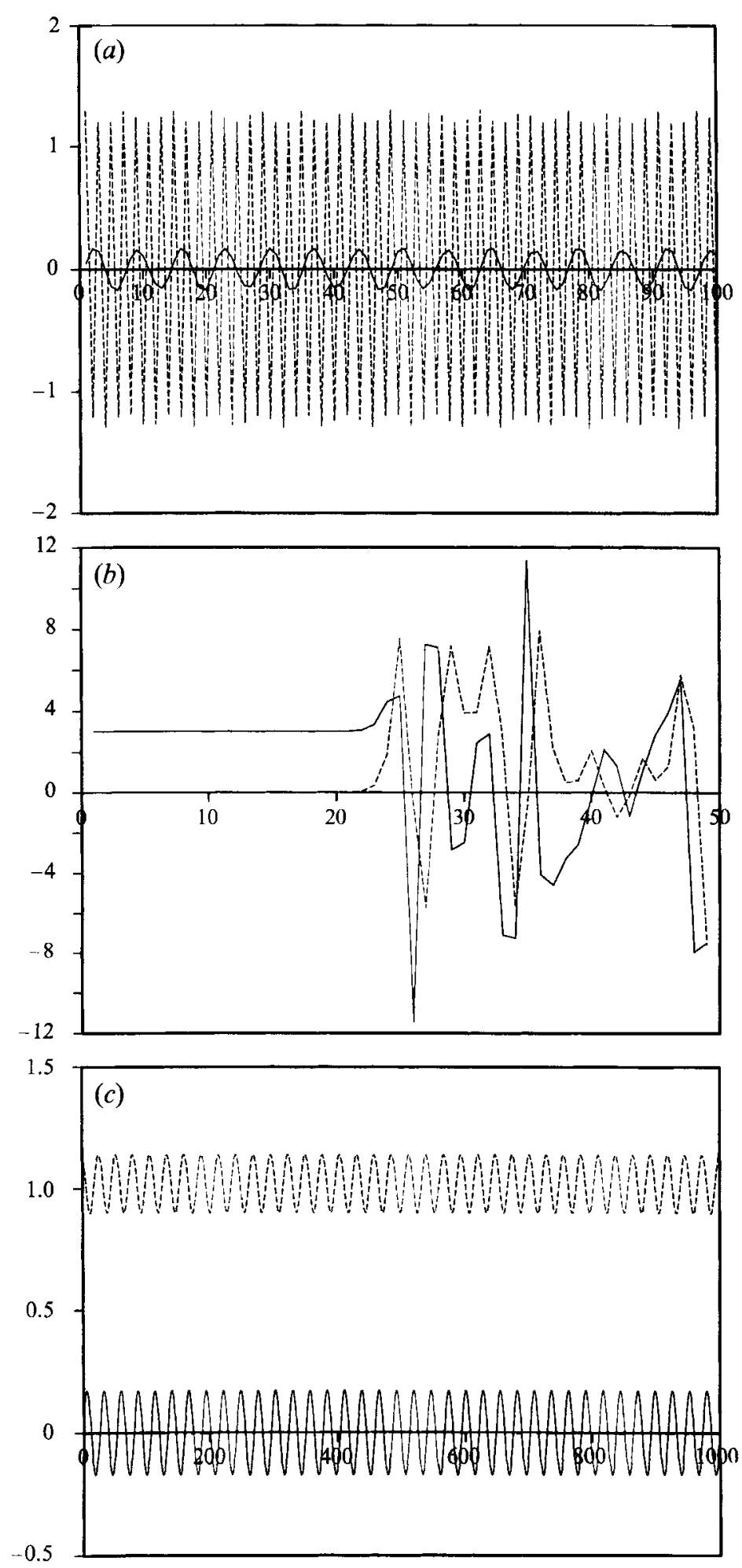

FIGURE $2(a-c)$. For caption see facing page. 


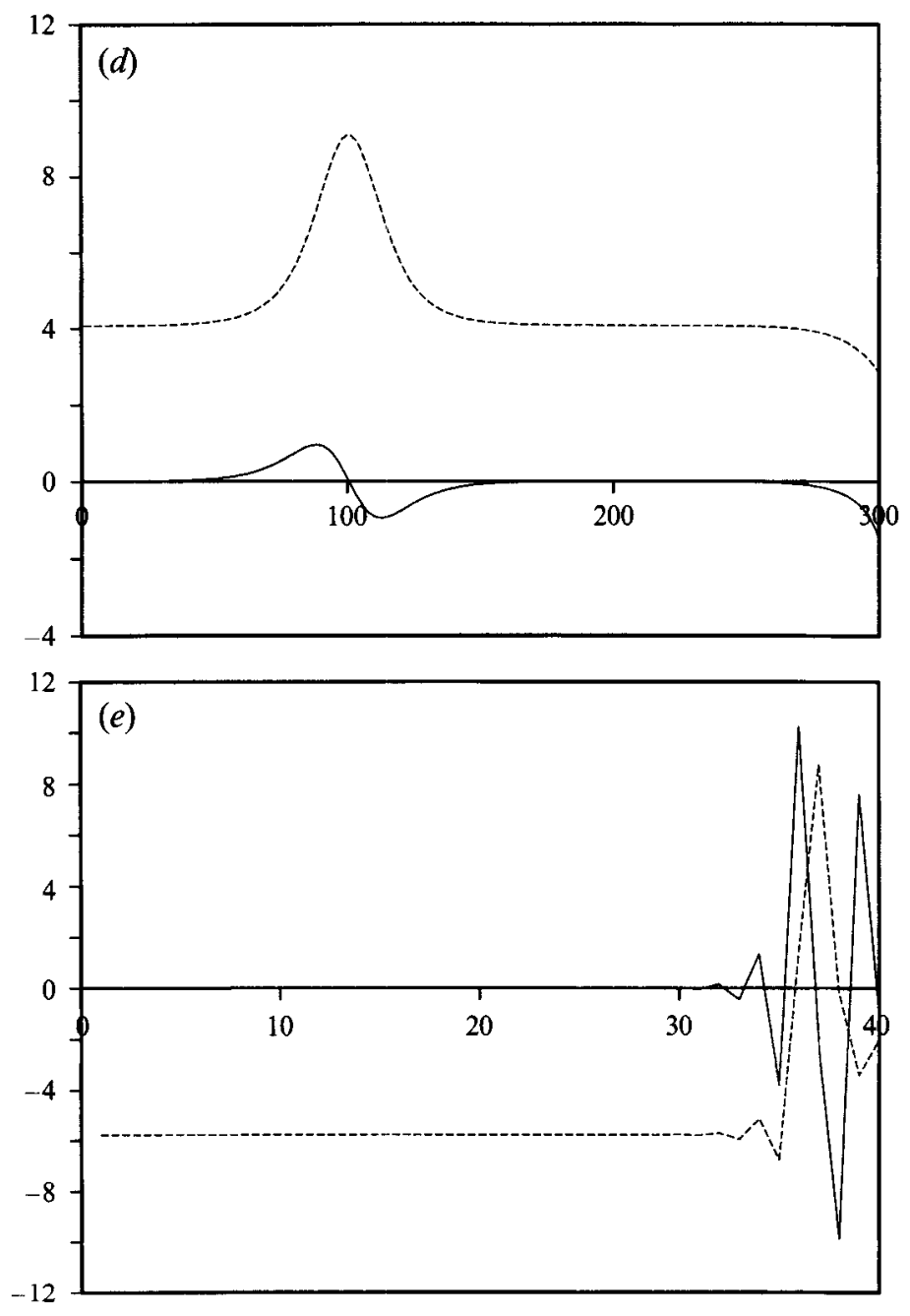

FIGURE 2. Solutions of the recurrence relations (7.1)-(7.3) with initial conditions in the neighbourhood of those for a periodic solution ( $\gamma$ continuous line, $\delta$ dashed line): $(a)$ stable with $I=0, L=1 ;(b)$ unstable with $I=0, L=1 ;(c)$ stable with $I=0.2728, L=10 ;(d)$ unstable with $I=0.5382, L=10$; (e) unstable with $I=2.9722, L=1$.

\section{(a) Periodic solutions}

Although it is easy to compute successive $\tilde{\gamma}, \tilde{\delta}$ from (7.1)-(7.3) the system of recurrence relations is nonlinear, and as such may have unpredictable behaviour. We first examine the possibility of periodic solutions. It is not clear what the definition of periodicity should be but, initially at least, we shall consider periodic solutions for which the distance $\sqrt{ } 2 s$ between successive humps is always the same. There is of course no reason why this distance itself should not be a periodic rather than a constant function.

With $\mu$ a constant it is not difficult to analyse the periodic solutions of (7.1)-(7.3). At any stage the point $(\tilde{\gamma}, \tilde{\delta})$ must lie on the ellipse $(7.3 b)$, and, when we update for the next stage it must also lie on (7.3a). Thus the (up to four) points of intersection of these ellipses determine the periodic solutions with equally spaced humps. When $I=0$ the points of intersection are $(0, \pm \mu),( \pm \mu, 0)$ and the values of $\mu$ for which the various combinations are possible form a sequence of eigenvalues. For example, it is easily 
verified that $(7.1)-(7.3)$ have the solution $\gamma=0, \delta=\mu, \tilde{\gamma}=0, \tilde{\delta}=\mu$ if $\left(s-2 y_{1}\right) / L=$ $p \pi$ for any integer $p$. Since $\mu$ is asymptotically small, and hence $s$ asymptotically large, for this theory to hold, strictly $p$ should be a large integer. In all there are four distinct possibilities, listed below, for periodic solutions with equal distances between humps. These are, where in each case $\mu$ may be replaced by $-\mu$,

(i) $\quad \gamma=0, \quad \delta=\mu, \quad \tilde{\gamma}=0, \quad \tilde{\delta}=(-1)^{p} \mu \quad$ with $\quad\left(s-2 y_{1}\right) / L=p \pi / 2$;

(ii) $\quad \gamma=\mu, \quad \delta=0, \quad \tilde{\gamma}=(-1)^{p+1} \mu, \quad \tilde{\delta}=0 \quad$ with $\quad\left(s-2 y_{0}\right) / L=p \pi / 2$;

(iii) $\quad \gamma=0, \quad \delta=\mu, \quad \tilde{\gamma}=(-1)^{p+1} \mu, \quad \tilde{\delta}=0 \quad$ with $\quad\left(s-y_{0}-y_{1}\right) / L=p \pi / 2 ;$

(iv) $\gamma=\mu, \quad \delta=0, \quad \tilde{\gamma}=0, \quad \tilde{\delta}=(-1)^{p} \mu \quad$ with $\left.\quad\left(s-y_{0}-y_{1}\right) / L=p \pi / 2.\right)$

Case (i) with $p$ even and case (ii) with $p$ odd are obviously immediately periodic (i.e. $\gamma=\tilde{\gamma}, \delta=\tilde{\delta}$ ) with period $\sqrt{ } 2 s$. If $p$ is odd in case (i) and even in case (ii) then two applications are required before $\gamma, \delta$ return to their original values and the period is $2^{3 / 2} s$. Cases (iii) and (iv) must be applied successively as a pair and the resulting period is $2^{5 / 2} s$.

When $I$, which we recall is proportional to the cross-flow, is non-zero the original equations (5.1) and hence the recurrence relations (7.1) lose their upstream/ downstream symmetry. However, if purely periodic solutions are sought it is sufficient to restrict attention to positive $I$ only because a change of sign of $N$ in (5.1) is equivalent to a change of sign of $x$. If $I$ is sufficiently small the ellipses again intersect in four points, namely $\left(0, \delta_{1}\right),\left(0, \delta_{2}\right),(\bar{\gamma}, \bar{\delta}),(-\bar{\gamma}, \bar{\delta})$ where

$$
\bar{\gamma}^{2}=\mu^{2}-I^{2} \tan ^{2} \frac{2}{L}\left(y_{0}-y_{1}\right), \quad \bar{\delta}=I \sec \frac{2}{L}\left(y_{0}-y_{1}\right)
$$

and $\delta_{1}, \delta_{2}$ are the roots of

$$
\left(\delta_{1,2}-I \cos \frac{2}{L}\left(y_{0}-y_{1}\right)\right)^{2}=\mu^{2}-I^{2} \sin ^{2} \frac{2}{L}\left(y_{0}-y_{1}\right) .
$$

Cases corresponding to (i)-(iv) of (7.4) are no longer all possible. One that does lead to a consistent eigenrelation for $\mu$ is that corresponding to (i) with $p$ even, i.e. $\gamma=\tilde{\gamma}=$ $0, \delta=\tilde{\delta}=\delta_{1,2}$. The eigenrelation for this is found from (7.1) to be

$$
\mu^{2} \sin ^{2} \frac{1}{L}\left(2 y_{1}-s\right)=I^{2} \sin ^{2} \frac{2}{L}\left(y_{0}-y_{1}\right)
$$

with the corresponding

$$
\delta=I \frac{\sin \frac{1}{L}\left(2 y_{0}-s\right)}{\sin \frac{1}{L}\left(2 y_{1}-s\right)} .
$$

Other combinations which might have been thought to have sufficient symmetry, for example $\gamma=\tilde{\gamma}=0$ with $\delta=\delta_{1}, \tilde{\delta}=\delta_{2}$ or $\gamma=\bar{\gamma}=-\tilde{\gamma}$ with $\delta=\bar{\delta}=\tilde{\delta}$ fail. The first clearly does not satisfy (7.3), while in the second the consequence of the lack of upstream/downstream symmetry is that although $\bar{\gamma}$ may be followed by $-\bar{\gamma}$ at the successive hump, it is not possible for the resulting $-\bar{\gamma}$ to be followed by $\bar{\gamma}$.

If $|I| \ll 1$, equation (7.7) reduces to

and (7.8) to

$$
\frac{1}{L}\left(s-2 y_{1}\right) \approx m \pi \pm \frac{I}{16} \exp \left(2 y_{1}+m \pi\right) \sin \frac{2}{L}\left(y_{0}-y_{1}\right)
$$

$$
\delta \approx \mp 16 \exp \left(-2 y_{1}-m \pi\right)
$$


for integral $m$, both of which, as $I \rightarrow 0$, reduce to case (i) of (7.4) with $p$ even. When $L \gg 1$, equation (7.7) requires $|I L| \ll 1$ and $\mu \approx I L / \log (16 / I L)$ with, from (7.8), $\delta \approx \mu$. To deduce these results, the asymptotic forms of $y_{0}, y_{1}$ in (A 1) have been used. Although (7.7) has solutions for $s$, and consequently for $\mu$, when $|I| \gg 1$, in such a situation $\mu=O(I)$ and although the recurrence relations have perfectly acceptable solutions in this regime these cannot be related to the solution of the original differential equations for which it was assumed that $\sqrt{ } 2 s$ is the distance between two maxima of $\widetilde{A}+\widetilde{B}$ and thus essentially positive. Hence, from (7.2), it follows that no solution with $\mu>16$ is of direct interest; strictly the validity of the analysis is $\mu \ll 1$.

It is simple to examine the linear stability of the periodic solutions (i)-(iv) in (7.4) with $I=0$ and of the periodic solution given by (7.7), (7.8) for non-zero $I$. If in (7.1)-(7.3) we write

$$
\gamma=\gamma_{0}+\epsilon_{1}, \quad \delta=\delta_{0}+\epsilon_{2}, \quad \mu=\mu_{0}+\delta \mu, \quad s=s_{0}+\delta s,
$$

where $\epsilon_{1}, \epsilon_{2}, \delta \mu, \delta s$ are small perturbations then, by linearization from (7.2), (7.3a), (7.1) successively we obtain

$$
\begin{gathered}
\delta s=-\delta \mu / \mu_{0}, \quad \mu_{0} \delta \mu=\left(\gamma_{0}-I\right) \epsilon_{1}+\delta_{0} \epsilon_{2}+\left(\left(\gamma_{0}-I\right) \epsilon_{2}+\delta_{0} \epsilon_{1}\right) \cos \left(Y_{0}-Y_{1}\right), \\
\begin{aligned}
& \tilde{\epsilon}_{1} \sin \left(Y_{0}-Y_{1}\right)=\epsilon_{1} \sin \left(Y_{0}+Y_{1}\right)+\epsilon_{2} \sin 2 Y_{1} \\
&\left.+\frac{2}{L} \frac{\delta \mu}{\mu_{0}}\left(\left(\gamma_{0}-I\right) \cos \left(Y_{0}+Y_{1}\right)+\delta_{0} \cos 2 Y_{1}\right)\right), \\
& \tilde{\epsilon}_{2} \sin \left(Y_{0}-Y_{1}\right)=\epsilon_{1} \sin 2 Y_{0}+\epsilon_{2} \sin \left(Y_{0}+Y_{1}\right) \\
&+\frac{2}{L} \frac{\delta \mu}{\mu_{0}}\left(\left(\gamma_{0}-I\right) \cos 2 Y_{0}+\delta_{0} \cos \left(Y_{0}+Y_{1}\right)\right) .
\end{aligned}
\end{gathered}
$$

Here $\tilde{\gamma}=\tilde{\gamma}_{0}+\tilde{\epsilon}_{1}, \tilde{\delta}=\tilde{\delta}_{0}+\tilde{\epsilon}_{2}, Y_{0}=\left(2 y_{0}-s_{0}\right) / L, Y_{1}=\left(2 y_{1}-s_{0}\right) / L$ and we must substitute for $\delta \mu / \mu_{0}$ from (7.12b).

Equations (7.13) are linear recurrence relations with constant coefficients which may be solved exactly. If we write them as

$$
u_{n+1}=a u_{n}+b v_{n}, \quad v_{n+1}=c u_{n}+d v_{n}
$$

then the condition for stability is that $|\lambda|<1$ where $\lambda$ satisfies

$$
\lambda^{2}-(a+d) \lambda+a d-b c=0 .
$$

It may be shown that each of cases (i)-(iv) of (7.4) leads to $a d-b c=1$ so that if the roots are real then one has modulus greater than unity. If the roots are complex they both have modulus unity, and the perturbation, if initially of sufficiently small amplitude, will oscillate about zero. In cases (iii) and (iv) the roots of (7.15) are real for all $y_{0}, y_{1}$, so the situations are unstable. In case (i) the roots are complex if

$$
\cos \left(Y_{0}-Y_{1}\right)\left(\cos \left(Y_{0}-Y_{1}\right)+L \sin \left(Y_{0}-Y_{1}\right)\right)<0,
$$

while in case (ii) the condition is

$$
\cos \left(Y_{0}-Y_{1}\right)\left(\cos \left(Y_{0}-Y_{1}\right)-L \sin \left(Y_{0}-Y_{1}\right)\right)<0 .
$$

It may be verified from tables 3 and 4 that (7.17) never holds but that (7.16) is satisfied for values of $L$ larger than about 0.6 . Thus only case (i) is stable. This is illustrated in figure 2(a) for a value of $L=1$ and initial conditions $\gamma=0.05, \delta=\mu_{0}+0.05$ and $s_{0}=$ $2 y_{1} / L+\frac{1}{2} \pi$ as an example of case (i) with $p=1$. With $p=0$ stability was maintained 
with even larger initial perturbations. However, an example of case (ii), again with $p=1$, is unstable owing to rounding error only; the instability is marked as can be seen in figure $2(b)$.

The values of $a, b, c, d$ in (7.14) for the periodic situation described by (7.7), (7.8) for non-zero $I$ may be shown to be

$$
\begin{aligned}
& a=d=\left(\sin \left(Y_{0}+Y_{1}\right)+\frac{2}{L} \cos Y_{0} \cos Y_{1}\right) / \sin \left(Y_{0}-Y_{1}\right), \\
& b=\left(\sin 2 Y_{1}+\frac{2}{L} \cos ^{2} Y_{1}\right) / \sin \left(Y_{0}-Y_{1}\right), \\
& c=\left(\sin 2 Y_{0}+\frac{2}{L} \cos ^{2} Y_{0}\right) / \sin \left(Y_{0}-Y_{1}\right),
\end{aligned}
$$

Again $a d-b c=1$ and the condition for the quadratic for $\lambda$ to have complex roots is now

$$
\cos Y_{0} \cos Y_{1}\left(\sin Y_{0} \sin Y_{1}+\frac{1}{L} \sin \left(Y_{0}+Y_{1}\right)+\frac{1}{L^{2}} \cos Y_{0} \cos Y_{1}\right)<0
$$

which reduces to (7.16), as it should, when $s_{0}$ is given by case (i) of (7.4) with $p$ even. It does not seem worthwhile to undertake an examination of the regions of the $(I, L)$ plane in which (7.19) is satisfied. When $L \gg 1$, however, (7.19) reduces to

$$
\left(2-s_{0}\right)\left(s_{0}-1\right)<0 \text {, }
$$

a condition which is easily checked.

Illustrations of the stability of the periodic solutions with $I \neq 0$ and $L=10$ are shown in figure $(2 c, d)$. The values of $s_{0}$ are $4 \log 2$ and $2 \log 2$ respectively, of which the former satisfies (7.20). The corresponding values of $I$ are, from (7.7), 0.2728 and 0.5382 and in figure $2(c)$ the initial values were taken as $\gamma=0.1, \delta=\delta_{0}+0.1$, but in figure $2(d)$ they were $\gamma=0.001, \delta=\delta_{0}+0.001$, for the appropriate $\delta_{0}$ from (7.8) in each case. The oscillations in figure $2(c)$ persist to large values of $n$ but in figure $2(d)$, where (7.20) is violated, the solution does not oscillate about the appropriate values even though the initial perturbations are far smaller. A similar picture to that in figure $2(c)$ is obtained with $s_{0}=\log 2, I=0.5142$, a situation in which (7.20) is again satisfied.

Figure 2(e) illustrates an example with a smaller value of $L$, namely $L=1$. Here $s_{0}=\log 2, I=2.9722$ but the inequality (7.19) is not satisfied; here the instability is due to rounding errors only. However, if $s_{0}=2 \log 2$ so that $I=2.0221$, the inequality is satisfied and extremely stable oscillations (not illustrated) are obtained.

Some comment may now be made on the effect of the parameters $L$ and $I$ on the stability of the periodic solutions of the recurrence relations. When $I=0$, only one type is stable and this requires $L>0.6$. Increasing $L$ seems to increase the degree of stability. However, when $I \neq 0$ this is not necessarily so as illustrated by the examples of figure 2. This is because of the complexity of condition (7.19). Increasing $I$ does not necessarily decrease the degree of stability either, although, as stated after (7.19), there are fewer periodic solutions as only those corresponding to case (i) of (7.4) with $p$ even are now possible.

\section{(b) Non-periodic solutions}

Solutions of the recurrence relations may easily be computed for any values of $L, I$ and initial values $\gamma, \delta$. The appropriate $\mu$ is calculated from (7.3a), $s$ from (7.2) and $\tilde{\gamma}, \tilde{\delta}$ from (7.1). Equation ( $7.3 b$ ) is then automatic. The next step is undertaken analogously 
after replacing $\gamma, \delta$ by the newly calculated $\tilde{\gamma}, \tilde{\delta}$. If, at any stage, the resulting $\mu$ from (7.3a) has $\mu>16$, then $s<0$ and the recurrence relations cannot describe a solution of the original differential equations (5.1). Indeed all solutions computed (except those that were a perturbation of case (i) in (7.4) above or of a stable situation with $I \neq 0$ ) sooner or later reached this stage and it seemed pointless to pursue them further.

The purpose of this section was to gain some feeling for what may be expected from the solutions of the differential equations (5.1). These equations have $\tilde{A} \equiv \tilde{B}$ as a solution, with a sech ${ }^{2}$ profile as in (6.1). In $\$ 6$ we used this solution as a basis to construct symmetric solutions in which the difference between $\tilde{A}$ and $\tilde{B}$ is small. Such solutions consist of an infinite number of humps and troughs, strictly an asymptotically large distance apart, and matching from hump to hump leads to difference equations for the relative heights and slopes of $\tilde{A}$ and $\tilde{B}$ at the top of successive humps. These equations depend on two parameters, $L$ and $N$. We have analysed the periodic solutions of these difference equations, i.e. those solutions that are periodic in that they lead to equidistantly spaced humps, and have examined the stability of these solutions. We have shown that, in the case of zero cross-flow, only in one type of these periodic solutions will perturbations remain in its neighbourhood when $L$ is sufficiently large, the other three types being unstable. With non-zero cross-flow there is one type of periodic solution, described by (7.7), (7.8) and its stability depends on both $L$ and $N$. When $L \gg 1$ but $s / L=O(1)$, the difference equations may be replaced by differential equations the solutions of which suggest that in this limit all solutions of the difference equations are periodic but not with constant $s$. Successive values of $s$ vary with $n$ but after a sufficiently large $n$, which can be estimated from the solution of the differential equations, the sequence of values of $s$ is repeated.

In the following section we return to the differential equations (5.1), and seek solutions in which $(\widetilde{A}-\widetilde{B})$ is not necessarily small. We use the results of this section to lead to solutions in which $\tilde{A}$ and $\widetilde{B}$ are periodic functions, predictions of the period being possible from the results of the asymptotic analysis.

\section{Periodic solutions of equations (5.1)}

The asymptotic analysis of the preceding sections with $|\tilde{A}-\tilde{B}| \ll 1$ shows that in this limit periodic solutions of (5.1) exist both when the cross-flow parameter $N$ is zero (as in (7.4)) and when it is small but non-zero (as in (7.7), (7.8)). It is reasonable to suppose that periodic solutions of (5.1) exist even when $|\tilde{A}-\widetilde{B}|$ is of order unity, and we now show, by obtaining such solutions explicitly by numerical means, that this is indeed so. These solutions are obtained by using the analytic results for small $|\tilde{A}-\tilde{B}|$ as an initial guess, for the unknown period for example. All solutions sought will have similar symmetry to those found in $\S \S 6,7$ and there is no suggestion that the study is exhaustive. For purposes of illustration we shall usually take $L=1$ except where $L \neq 1$ is of particular interest.

We first consider the analogue of cases (i) and (ii) of (7.4). From (6.15), (6.17) and (6.19) we find that, for each, (6.20) reduces to $h_{n}=p \pi / 2$. Examination of (6.14) then shows that, if $p$ is even, $\tilde{A}=\widetilde{B}$ at $x=t_{n}$, the base of the trough, but if $p$ is odd then $\tilde{A}-\widetilde{B}= \pm \mu_{n} \cos \left(\lambda_{n}\left(x-t_{n}\right) / L\right)$ which, together with (6.13), implies that either $\tilde{A}$ or $\widetilde{B}$ vanishes at $x=t_{n}$. In cases (iii) and (iv) we obtain $h_{n}=\frac{1}{2} p \pi \pm\left(y_{0}-y_{1}\right) / L$ respectively. In both cases (i) and (ii) $\widetilde{A}+\widetilde{B}$ is even about the trough and the hump; if $p$ is even then $\tilde{A}-\tilde{B}$ is odd about the trough but is even when $p$ is odd. Case (i) has $\tilde{A}-\tilde{B}$ odd about the humps while in case (ii) it is even. Cases (iii) and (iv) do not have so much symmetry although $\widetilde{A}+\widetilde{B}$ is even about both trough and hump. 


\begin{tabular}{|c|c|c|c|c|c|c|}
\hline & $p$ & $s$ & $\tilde{a}_{0}($ a.e. $)$ & $\tilde{b}_{2}($ a.e. $)$ & $\tilde{a}_{0}(\mathrm{num})$ & $\tilde{b}_{2}($ num $)$ \\
\hline (a) & $\begin{array}{l}1 \\
3 \\
5 \\
7\end{array}$ & $\begin{array}{r}2.5554 \\
5.6970 \\
8.8386 \\
11.9801\end{array}$ & $\begin{array}{l}4.0571 \\
0.8714 \\
0.0906 \\
0.0072\end{array}$ & $\begin{array}{r}26.4933 \\
28.2816 \\
7.0807 \\
1.0328\end{array}$ & $\begin{array}{l}3.4427 \\
0.8762 \\
0.0906 \\
0.0072\end{array}$ & $\begin{array}{r}10.1554 \\
27.2290 \\
6.9580 \\
1.0155\end{array}$ \\
\hline (b) & $\begin{array}{l}p \\
2 \\
4 \\
6\end{array}$ & $\begin{array}{c}s \\
4.1262 \\
7.2678 \\
10.4093\end{array}$ & $\begin{array}{l}\tilde{x}_{0}(\text { a.e. }) \\
1.0994 \\
0.1471 \\
0.0131\end{array}$ & $\begin{array}{c}\tilde{y}_{1} \text { (a.e.) } \\
4.5366 \\
1.0712 \\
0.1360\end{array}$ & $\begin{array}{c}\tilde{x}_{0} \text { (num) } \\
1.1442 \\
0.1476 \\
0.0131\end{array}$ & $\begin{array}{c}\tilde{y}_{1} \text { (num) } \\
4.4047 \\
1.0700 \\
0.1360\end{array}$ \\
\hline (c) & $\begin{array}{l}p \\
3 \\
5 \\
7\end{array}$ & $\begin{array}{c}s \\
4.8128 \\
7.9544 \\
11.0960\end{array}$ & $\begin{array}{l}\tilde{a}_{0} \text { (a.e.) } \\
1.5056 \\
0.1777 \\
0.0150\end{array}$ & $\begin{array}{r}\tilde{b}_{2} \text { (a.e.) } \\
34.8742 \\
11.2452 \\
1.8500\end{array}$ & $\begin{array}{c}\tilde{a}_{0} \text { (num) } \\
1.5338 \\
0.1778 \\
0.0150\end{array}$ & $\begin{array}{c}\tilde{b}_{2} \text { (num) } \\
32.7319 \\
11.0378 \\
1.8098\end{array}$ \\
\hline (d) & $\begin{array}{l}p \\
2 \\
4 \\
6\end{array}$ & $\begin{array}{c}s \\
3.2420 \\
6.3836 \\
9.5252\end{array}$ & $\begin{array}{l}\tilde{x}_{\mathbf{0}} \text { (a.e.) } \\
1.6432 \\
0.2753 \\
0.0265\end{array}$ & $\begin{array}{r}\tilde{y}_{1} \text { (a.e.) } \\
5.3274 \\
1.7575 \\
0.2523\end{array}$ & $\begin{array}{c}\tilde{x}_{0} \text { (num) } \\
2.1070 \\
0.2766 \\
0.0265\end{array}$ & $\begin{array}{c}\tilde{y}_{1} \text { (num) } \\
5.6899 \\
1.7534 \\
0.2523\end{array}$ \\
\hline
\end{tabular}

TABLE 1(a). Case (i), $p$ odd: predicted (cols. 3, 4) and calculated (cols. 5, 6) values of $\tilde{a}_{0}, \tilde{b}_{2}$. (b) Case (i) $p$ even: predicted (cols. 3,4$)$ and calculated (cols. 5,6) values of $\tilde{x}_{0}, \tilde{y}_{1} .(c)$ Case (ii) $p$ odd: predicted and calculated values of $\tilde{a}_{0}, \tilde{b}_{2}$. (d) Case (ii) $p$ even: predicted and calculated values of $\tilde{x}_{0}, \tilde{y}_{1}$.

We aim to compute the lowest modes corresponding to cases (i) and (ii) of (7.4). The eigenvalue is $\sqrt{ } 2^{1 / 2} s$, the distance between the humps, and first approximations to $s$ are given by (7.4) although strictly the formulae there hold only for $s \gg 1$. It emerges that the lowest possible $s$ in each situation leads to the simplest form of eigenfunction with the fewest oscillations in $\tilde{A}-\tilde{B}$. The most awkward cases to compute are those of (i) and (ii) with $p$ odd, since then either $\tilde{A}$ or $\tilde{B}$ vanishes at the base of the trough so that (5.1) have a regular singular point. To compute the periodic solutions we first scale the equations so that the distance between trough and succeeding hump is unity. The scaling that takes the interval $\left(0,2^{-1 / 2} s\right)$ into $(0,1)$ and leaves $(5.1)$ unaltered when $N=0$, but replaces $N$ by $2^{-1 / 2} S N$ otherwise, is

$$
(x, \tilde{A}, \tilde{B}, N) \rightarrow\left(2^{-1 / 2} s \tilde{x}, 2 s^{-2} \tilde{\tilde{A}}, 2 s^{-2} \tilde{\widetilde{B}}, 2^{1 / 2} s^{-1} \tilde{N}\right) .
$$

Integration is then initiated from the lowest point of the trough ( $\tilde{x}=0$ say) with a target to be attained at the hump $\tilde{x}=1$. The target vector is of length 2 and there are two free parameters at $\tilde{x}=0$. Specifically these are, for the respective cases,

(i) target:

$$
\begin{aligned}
& \tilde{\widetilde{A}}(1)=\tilde{\widetilde{B}}(1), \quad \tilde{\widetilde{A}}^{\prime}(1)+\tilde{\widetilde{B}}^{\prime}(1)=0 ; \\
& \widetilde{\widetilde{A}}^{\prime}(1)=\widetilde{\widetilde{B}}^{\prime}(1)=0 \text {. }
\end{aligned}
$$

In both cases (i) and (ii) $\tilde{\widetilde{A}}+\widetilde{\widetilde{B}}$ is even about the hump and in case (i) $\tilde{\widetilde{A}}-\tilde{\tilde{B}}$ is odd but is even in case (ii). The free parameters of $\tilde{x}=0$ follow from the fact that in all cases $\widetilde{\widetilde{A}}+\widetilde{B}$ is even about the trough, but if $p$ is odd $\widetilde{\widetilde{A}}-\widetilde{\widetilde{B}}$ is even, and conversely. If, when $p$ is odd, we denote the free parameters $\widetilde{\widetilde{A}}(0),{\widetilde{\tilde{B}^{\prime \prime}}}^{\prime}(0)$ by $\tilde{a}_{0}, \tilde{b}_{2}$ (on the assumption that it is $\tilde{B}$ that vanishes at the trough) then when $L=1$, for small $\tilde{x}$

$$
\begin{gathered}
\tilde{\tilde{A}}(\tilde{x})=\tilde{a}_{0}+\tilde{b}_{2}\left(\tilde{b}_{2}-\tilde{a}_{0}^{2}\right) \tilde{x}^{4} / 2 a_{0}+O\left(\tilde{x}^{6}\right), \\
\tilde{\tilde{B}}(\tilde{x})=\tilde{b}_{2} \tilde{x}^{2} / 2-\tilde{a}_{0} \tilde{b}_{2} \tilde{x}^{4} / 12+O\left(\tilde{x}^{6}\right) .
\end{gathered}
$$


On vortex/wave interactions. Part 1
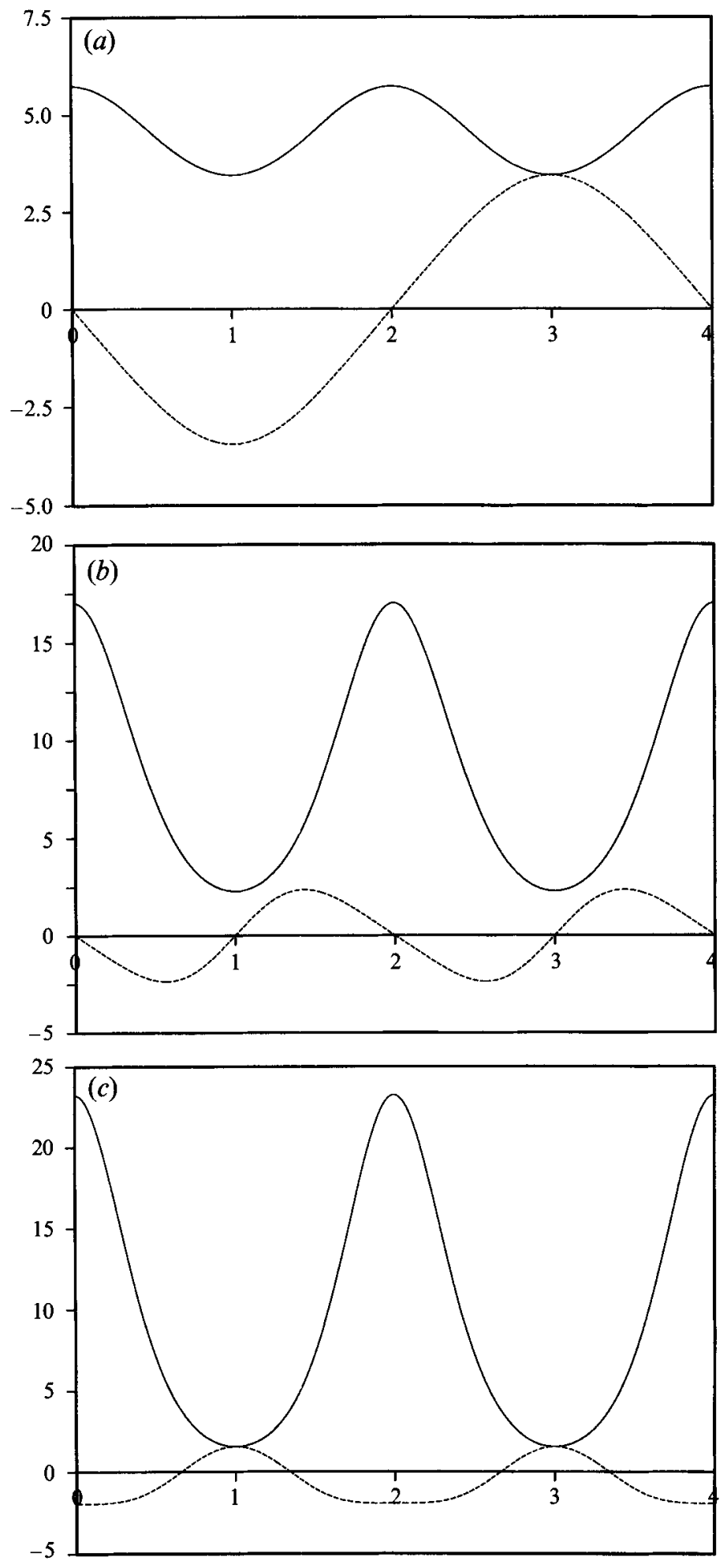

FIGURE $3(a-c)$. For caption see page 123. 

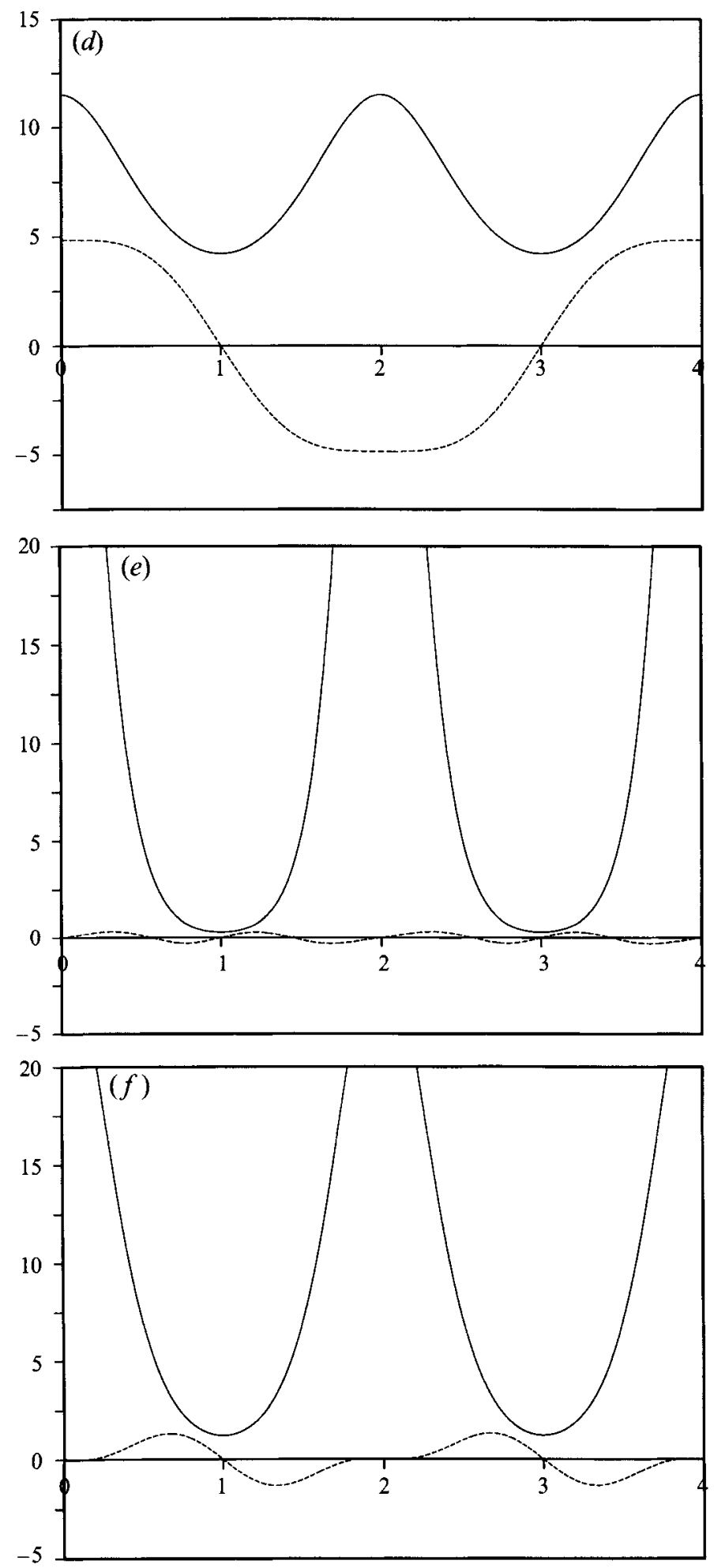

FIGURE $3(d-f)$. For caption see facing page. 


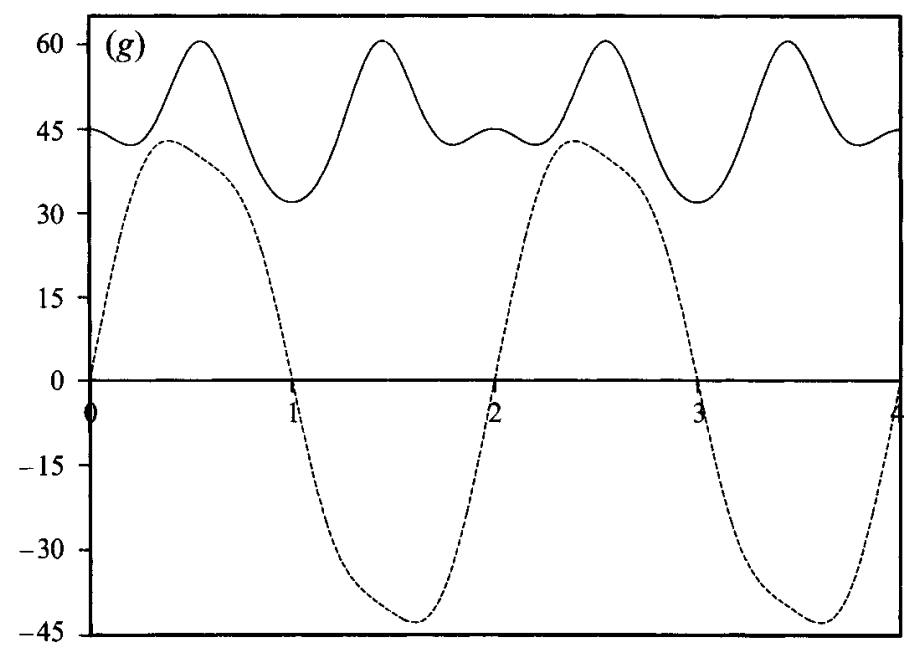

FIGURE 3. $(a)-(d)$. The lowest periodic mode of table $1(a-d)$ respectively $(\tilde{\tilde{A}}+\tilde{\tilde{B}}$ continuous line, $\tilde{\tilde{A}}-\tilde{\tilde{B}}$ dashed line, abscissa $\tilde{x}$ ). $\tilde{N}=0, L=1$. (e) The second periodic mode of table $1(b)$ on the same scale as figure $3(b) . \tilde{N}=0, L=1$. (f) The change from a mode of the first type to one of the second type near $\tilde{N}=0.075$. Again $L=1$. $(\mathrm{g})$ An additional periodic mode with $N=0, L=1$.

When $p$ is even the free parameters are $\tilde{x}_{0}, \tilde{y}_{1}$ where

$$
\tilde{x}_{0}=\tilde{\widetilde{A}}(0)(=\tilde{\widetilde{B}}(0)) \quad \text { and } \quad \tilde{y}_{1}=\tilde{\widetilde{A}}^{\prime}(0)\left(=\left(\tilde{\widetilde{B}}^{\prime}(0)\right) .\right.
$$

With $L=1$ we have from tables 3 and 4 that $y_{0}=0.0502, y_{1}=0.4923$. We now use cases (i) and (ii) of (7.4) to predict the values of $s$ for the first few values of $p$. This will enable the values of $\tilde{a}_{0}, \tilde{b}_{2}, \tilde{x}_{0}, \tilde{y}_{1}$ to be predicted as initial guesses for the Newton iteration for the target in the respective cases. The predictions are made as follows. Once $s$ is known, $\mu$ follows from $(7.2)$, and then $\widetilde{A}(0)=\mu s^{2} / 2$ and $\widetilde{B^{\prime \prime}}(0)=\mu s^{4} / 2$ from (6.13), (6.14) giving $\tilde{a}_{0}$ and $\tilde{b}_{2}$ in the cases when $p$ is odd. When $p$ is even, it follows from (6.13), (6.14) that $\tilde{x}_{0}=\mu s^{2} / 4$ and $\tilde{y}_{1}=\mu s^{3} / 4$. In table $1(a-d)$ we give the first few values of $s, \tilde{a}_{0}, \tilde{b}_{2}, \tilde{x}_{0}, \tilde{y}_{1}$ as predicted by the asymptotic formulae and by the Runge-Kutta Newtonian method. Table $1(a, b)$ shows case (i) with $p$ odd and even respectively and table $1(c, d)$ shows case (ii) analogously.

We see from the table that the asymptotic formulae increase dramatically in

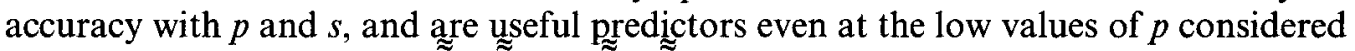
here. Figure $3(a-d)$ shows $\widetilde{\widetilde{A}}+\widetilde{\widetilde{B}}$ and $\widetilde{\tilde{A}}-\widetilde{\widetilde{B}}$ for the smallest value of $p$ in each of tables $1(a)-1(d)$. For convenience we have chosen to plot the figure so that $\tilde{x}=0,2,4$ correspond to humps of the asymptotic theory while $\tilde{x}=1,3$ correspond to troughs. In figure $3(b, c)$ the period is 2 , and in $3(a, d)$ it is 4 . The higher modes increasingly resemble the asymptotic expansion. With a period scaled to be of order unity the heights of the humps increase as $s^{2}$ (see (8.1)) and they resemble more closely sech ${ }^{2}$ profiles; in addition the number of oscillations of $\widetilde{\tilde{A}}-\widetilde{\tilde{B}}$ between hump and trough increases. As an illustration of this in figure 3(e) we plot the second mode of table 1(b) on the same scale as the first mode in figure $3(b)$. It contains an extra oscillation of $\widetilde{\tilde{A}}-\widetilde{\widetilde{B}}$ between hump and trough and the maximum value of $\tilde{\tilde{A}}+\widetilde{\widetilde{B}}$ is approximately 52.8 .

There are also periodic solutions of (5.1) when $N$ is non-zero. Although it is possible to scale $N$ out of the equations it is not possible to scale out the period as well. Thus we retain $N$ and as before scale solutions with unit distance between hump and trough. The asymptotic analysis of $\$ \S 6,7$ leads to one type of periodic solution when both 
$|\tilde{A}-\tilde{B}|$ and $N$ are small. This corresponds to type (i) with $p$ even of (7.4), and (7.9) shows that for non-zero $N$ there are two such solutions. In table 2(a) we illustrate the periodic solutions obtained from perturbing the leading mode in table $1(b)$ but with $\tilde{y}_{1}=-4.407$. We see that as $\tilde{x}_{0}$ decreases, $\tilde{N}$ reaches a maximum (of 0.076 ) and decreases again to zero at which value the second mode of table $1(b)$ is attained. The quantities $\tilde{\tilde{x}}_{0}, \tilde{\tilde{y}}_{1}$ denote the values of $\tilde{A}$ and of $\tilde{A}^{\prime}$ at the hump. The change from a mode of the first type to that of the second type is illustrated in figure $3(f)$ in which $\tilde{N}=0.075$. This may be compared with figures $3(b)$ and $3(e)$.

A bound to $\tilde{N}$ is also obtained if the leading mode of table $1(b)$ with $\tilde{y}_{1}=4.4047$ is perturbed. The results of so doing are shown in table $2(b)$. As $\tilde{\tilde{N}}$ increases $\tilde{x}_{0}$ increases and $\tilde{y}_{1}$ decreases until the periodic solution degenerates to $\tilde{A}=\tilde{B}=8 \tilde{N}^{2}$, the exact solution of (5.1) noted in $\S 5$. This happens at $\tilde{N}=0.575$. If $\tilde{x}_{0}$ is increased beyond a value of 2.645 , the hump and the trough are interchanged and $\tilde{N}$ decreases to zero again. The value of $\tilde{N}$ at which the maximum occurs can be calculated by linearly perturbing the constant solution of (5.1). The perturbations are proportional to $\mathrm{e}^{ \pm \mathrm{i} \lambda \hat{N} x}$ where $\lambda^{2}=16\left(1 \pm \frac{1}{2} \sqrt{ } 3\right)$, and thus are of period 2, as are those solutions we have been seeking of the nonlinear equations, when $|\lambda| \tilde{N}$ is a multiple of $\pi$. The lowest value of $\tilde{N}$ from this argument is 0.57495 and at this value the solutions of the nonlinear equations reduce to linear perturbations of the constant solutions. The constant solutions exist for all values of $\widetilde{N} \neq 0$ (and $L$ ) and can be shown to be neutrally stable, but in general the periods of the oscillations are not equal to 2 . The significance of the higher values of $N$ at which the period of small oscillations is 2 has not been investigated.

The cut-off values of $\tilde{N}$ evident in table 2 do not imply that periodic solutions do not exist at larger values of $\tilde{N}$. The scaling properties of (5.1) result in the equivalence of a value $N_{0}$ of $\tilde{N}$ and a period of 2 , and a value $M_{0}$ of $\tilde{N}$ and a period $2 N_{0} / M_{0}$. Thus as $\tilde{N}$ increases the period decreases and the amplitudes of the eigenfunctions increase as $\left(M_{0} / N_{0}\right)^{2}$.

The development of the higher modes in table $2(b)$ as $\tilde{N}$ increases has not been pursued, nor is it suggested that all periodic solutions with $\widetilde{N} \neq 0$ may be obtained by perturbing those of case (i) of (7.4) with $p$ even as in (7.9). The reason for success in this situation is that (5.1) are invariant under a change of sign of $x$ provided $\tilde{A}$ and $\tilde{B}$ are also interchanged so a symmetric cross-over with $\tilde{A}-\tilde{B}$ odd about both hump and trough is acceptable. The other cases of (7.4) do not have this property

In each of tables $1(a)-1(d)$ successive modes have an extra zero of $\tilde{\tilde{A}}-\tilde{\widetilde{B}}$ between trough and hump. 'Higher' modes also exist in which $\widetilde{A}+\widetilde{B}$ has additional stationary points between trough and hump. Figure $3(g)$ for example, has $\widetilde{\widetilde{A}}-\tilde{\widetilde{B}}$ odd and $\tilde{\widetilde{A}}+\widetilde{B}$ even about both trough and hump with no additional zero of $\widetilde{\widetilde{A}}-\widetilde{\widetilde{B}}$ than in figure $3(b)$ but a more complex $\tilde{A}+\widetilde{B}$. In a sense it appears to be a 'second' mode, but clearly differs from that of figure $3(b)$. We have no analytic formula for the predictions of modes such as those of figure $3(\mathrm{~g})$ but their development with $\widetilde{N}$ could be examined, although we shall not do so here.

The periodic solutions computed here with $L=1$ may be traced as $L$ varies. Some such solutions have been obtained in the case of $N=0$. In most cases it was found that the solutions persisted as $L$ increased but some disappeared as $L$ decreased. For example, the simplest periodic solution, that with one oscillation of $\tilde{A}-\tilde{B}$ between the maxima of $\widetilde{A}+\widetilde{B}$, came to an end at $L^{2}=\frac{1}{3}$. This phenomenon indicated the presence of multiple 'lowest mode' solutions for $L^{2}>\frac{1}{3}$ and indeed such were found. However we shall not pursue this question of non-uniqueness. 


\begin{tabular}{|c|c|c|c|c|c|}
\hline & $\tilde{N}$ & $\tilde{x}_{0}$ & $\tilde{y}_{1}$ & $\tilde{\tilde{x}}_{0}$ & $\tilde{\tilde{y}}_{1}$ \\
\hline \multirow[t]{10}{*}{ (a) } & 0 & 1.1442 & -4.4047 & 8.5013 & 2.7584 \\
\hline & 0.02 & 1.0775 & -4.3306 & 8.9716 & 2.4717 \\
\hline & 0.04 & 0.9984 & -4.2110 & 9.5665 & 2.0889 \\
\hline & 0.06 & 0.8936 & -4.0029 & 10.4303 & 1.5229 \\
\hline & 0.076 & 0.6372 & -3.2755 & 13.1156 & 0.0000 \\
\hline & 0.06 & 0.4602 & -2.6055 & 15.8311 & -0.9053 \\
\hline & 0.04 & 0.3486 & -2.1142 & 18.2666 & -1.2135 \\
\hline & 0.02 & 0.2593 & -1.6544 & 21.1488 & -1.1741 \\
\hline & 0.00 & 0.1476 & -1.0700 & 26.4114 & -0.6498 \\
\hline & $\tilde{N}$ & $\tilde{x}_{0}$ & $\tilde{y}_{1}$ & $\tilde{\tilde{x}}_{0}$ & $\tilde{\tilde{y}}_{1}$ \\
\hline \multirow[t]{8}{*}{ (b) } & 0 & 1.1442 & 4.4047 & 8.5013 & -2.7584 \\
\hline & 0.1 & 1.3948 & 4.4404 & 6.9109 & -3.49007 \\
\hline & 0.2 & 1.5856 & 4.1692 & 5.8341 & -3.6165 \\
\hline & 0.3 & 1.7541 & 3.6871 & 4.9711 & -3.3828 \\
\hline & 0.4 & 1.9244 & 2.9932 & 4.2171 & -2.8470 \\
\hline & 0.5 & 2.1419 & 1.9719 & 3.4909 & -1.9248 \\
\hline & 0.575 & 2.6450 & 0 & 2.6450 & 0 \\
\hline & 0.6 & $8 \tilde{N}^{2}$ & 0 & $8 \tilde{N}^{2}$ & 0 \\
\hline
\end{tabular}

TABLE 2(a). The development of the first of the lowest modes with $\tilde{N}$. At the maximum value of $\tilde{N}$ it becomes a higher mode. $(b)$ The development of the second of the lowest modes with $\tilde{N}$. At the critical value of $\tilde{N}$, the functions become constant.

\section{Representative solutions at moderate values of $L$ and $N$}

In this section we present a few numerical solutions of (5.1) with fairly arbitrary initial conditions. In figure $4(a)$ are plotted $\tilde{A}+\tilde{B}$ and $\tilde{A}-\tilde{B}$ calculated with $L=1$, $N=0$ and

$$
\tilde{A}(0)=1.0, \quad \tilde{B}(0)=2.0, \quad \tilde{A}^{\prime}(0)=0.5, \quad \tilde{B}^{\prime}(0)=-0.5 .
$$

A NAG routine with self-adjusting step length was used so that the evidently very small values of $\widetilde{A}$ or $\widetilde{B}$ could be accommodated, although the same results could be achieved with a constant step length of $10^{-3} / 16$ and a conventional fourth-order Runge-Kutta scheme. A notable feature of figure $4(a)$ is the boundedness of both amplitude functions $\tilde{A}$ and $\widetilde{B}$. Indeed, in this example, the initial values are barely exceeded. Similar behaviour resulted with other choices of initial conditions.

Solutions with non-zero cross-flow were then sought. For this illustration we took $N=1$ and the initial conditions to be as in (9.1). Figure $4(b)$ shows $\tilde{A}+\tilde{B}$ and $\tilde{A}-\tilde{B}$ derived from the NAG routine with a tolerance of $10^{-10}$. This solution is reproducible (either by changing the tolerance in the NAG routine or by using the fixed-step-length Runge-Kutta routine) as far as $x$ approximately equal to 14.2, after which graphical inconsistencies may be detected. There is no doubt, however, that there are dramatic increases in amplitude in the neighbourhood of $x=2,4,14$ all of which follow a near zero of $\tilde{A}$ or $\tilde{B}$. We have no quantitative theoretical explanation of this phenomenon, which occurs also for a change of sign of $N$ and variations on the initial conditions (9.1), and only note that there seems to be a high degree of instability. There are, however, solutions with $L=N=1$, the behaviour of which we are able to understand. One of these is illustrated in figure $4(c)$ for which

$$
\tilde{A}(0)=8.5, \quad \tilde{B}(0)=6.5, \quad \tilde{A}^{\prime}(0)=0.5, \quad \tilde{B}^{\prime}(0)=0.5 .
$$

Here $\tilde{A}+\tilde{B}$ and $\tilde{A}-\tilde{B}$ are plotted and we see that both $\tilde{A}$ and $\tilde{B}$ oscillate about the 

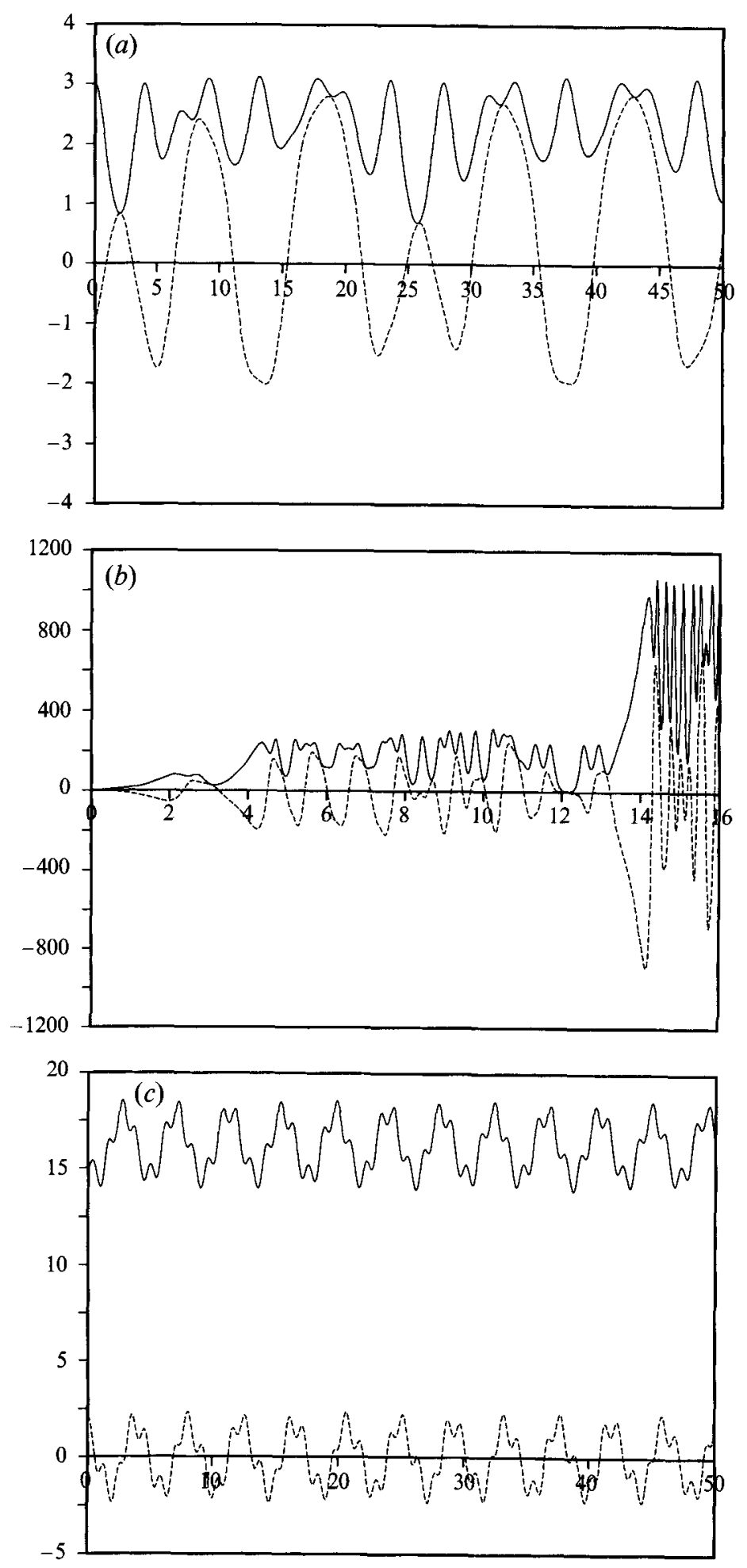

FIGURE 4. (a) Non-periodic solutions of equations (5.1) with initial conditions (9.1). $(\tilde{A}+\tilde{B}$ continuous line, $\tilde{A}-\tilde{B}$ dashed line, abscissa $x$.) $(a) N=0, L=1$; (b) $N=1, L=1$. (c) Oscillations about the exact solution $\tilde{A}=\widetilde{B}=8$ with $N=L=1$. 
value 8 which, as noted in $\S 5$ and again in $\S 8$ is an exact solution of (5.1). In $\S 8$ we stated that perturbations to this solution are proportional to $\mathrm{e}^{ \pm \mathrm{i} \lambda x}$ where $\lambda^{2}=$ $16\left(1 \pm \frac{1}{2} \sqrt{ } 3\right)$, and a weakly nonlinear theory applied to one such mode leads to a Stuart-Landau equation for the amplitude of the form

$$
\frac{\mathrm{d} A}{\mathrm{~d} X}=\mathrm{i} A|A|^{2}
$$

for appropriately scaled $x$. The solutions of (9.3) have $|A|$ constant and are purely oscillatory. This behaviour is consistent with that in figure $4(c)$ although the solution there is likely to consist of a combination of modes. The irrational values of $\lambda$ mean that there will be no resonances between these modes.

At more extreme values of $L$ it is possible to compute reproducible solutions with $N=1$ and the initial conditions (9.1). Figure 5(a) shows $\widetilde{A}+\widetilde{B}$ and $\widetilde{A}-\widetilde{B}$ with $N=1$ and $L=0.005$ while figure $5(b)$ has $L=20$. In both cases the solutions are remarkably periodic. Figure $5(c, d)$ shows the detailed structure of the rapid oscillations of $\tilde{A}-\widetilde{B}$ in figure $5(a)$ and $\widetilde{A}+\widetilde{B}$ in figure $5(b)$. Also evident, as expected, is the lack of upstream/downstream symmetry unless $\tilde{A}$ and $\widetilde{B}$ are also interchanged. Similar periodicity or quasi-periodicity is also evident at extreme values of $L$ when the crossflow is zero.

\section{Summary and conclusions}

In this study we have obtained equations for the amplitudes of two Rayleigh waves in vortex-wave interaction with a cross-flow. Although equally inclined to the mainstream the waves may be of unequal amplitude, and indeed must be in the case of non-zero cross-flow. The investigation centres on the question of the existence of self-perpetuating solutions at large distances downstream. Solutions that decay with $x$ or become singular at finite values of $x$ are not of interest here, and the parameter values, in particular $M<0$ in (4.3) and $A \lambda>0$ preceding (5.1), are chosen to exclude them. Since $x \gg 1$, the conditions at $x=-\infty$ are not expected to be relevant, and equations (4.7) have been replaced by the autonomous system (5.1); it is anticipated that this will hold in the neighbourhood of a large fixed value $X_{0}$ of $x$.

Equations (5.1) contain the two parameters $L$ and $N$. Here $N$ is a measure of the amount of cross-flow and was zero in SBB where only solutions with equal amplitudes $\tilde{A}=\widetilde{B}$ were considered; this particular equal-amplitude solution does not exist when $N \neq 0$. The parameter $L$ measures the phase difference of the $x$-derivative and nonlinear terms in the original equations (3.16). If $L \gg 1$, then $A$ and $C$ in (3.16) are both real (and of the same sign), but if $L \ll 1$, the ratio $C / A$ is purely imaginary.

Attention was focused on the periodic solutions of (5.1) on the grounds that they would correspond to self-sustaining solutions of the original equations. It was not clear $a b$ initio that such solutions would exist, but several families were constructed using an asymptotic technique. These solutions had approximately equal values of amplitudes $\widetilde{A}$ and $\widetilde{B}$ and necessarily small (or zero) values of $N$. They were characterized by a hump-trough sequence pattern, a hump representing an interval of intense wave activity and a trough one of quiescence with consequent vortex adjustments. Conditions at successive humps are related by difference equations studied in $\$ 6$. Periodic solutions of these are identified and used as aids to constructing periodic solutions for finite values of $|\tilde{A}-\tilde{B}|$ by numerical means. For illustration most of the examples computed have $L=1$, and it seems likely (see below) that periodic solutions 

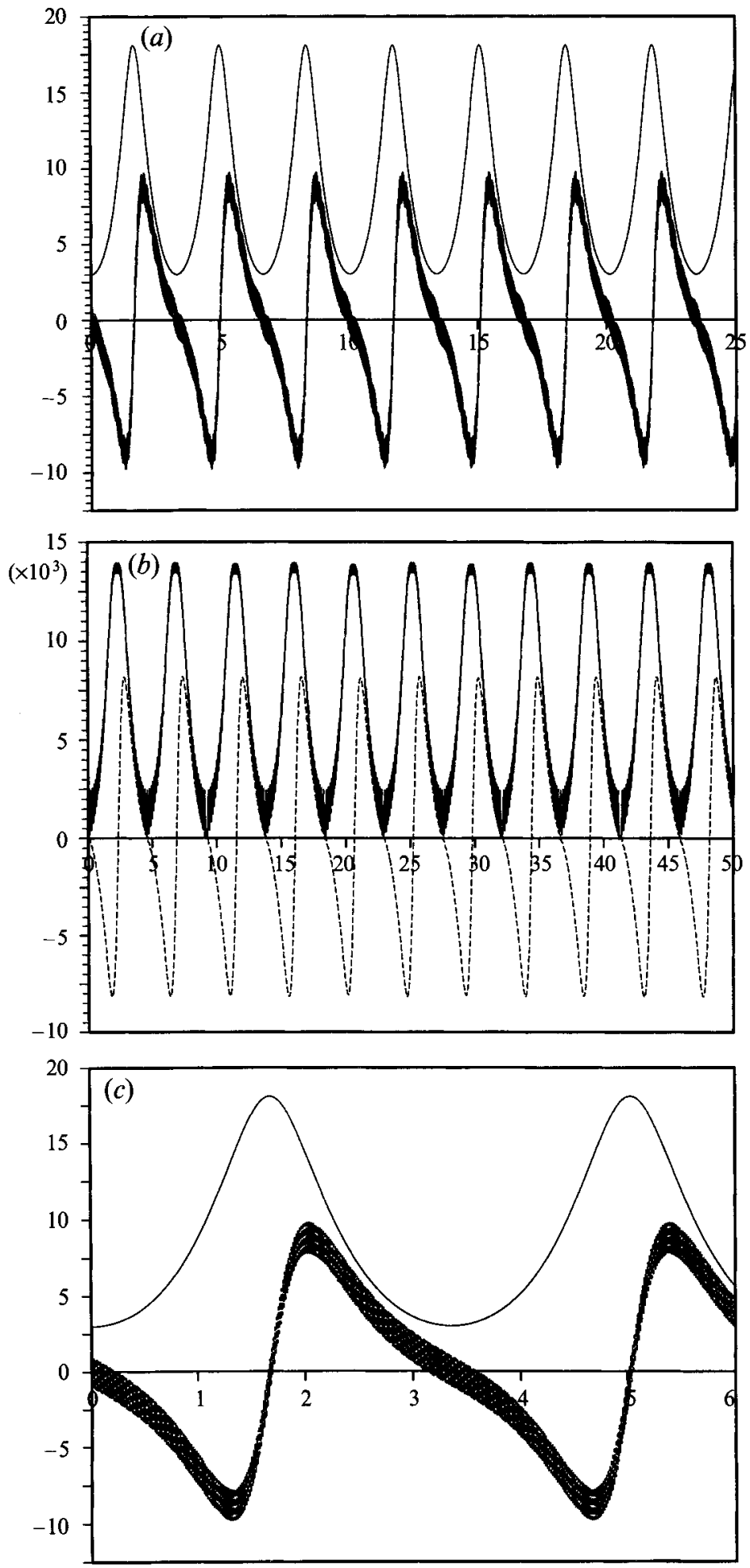

FIGURE $5(a-c)$. For caption see facing page. 


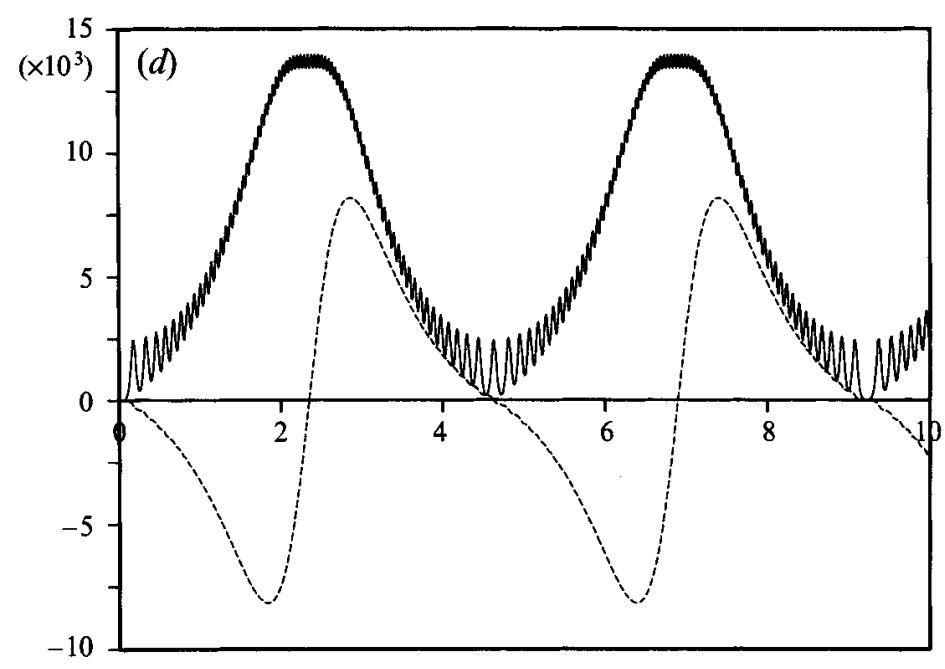

FIGURE 5. (a) Solutions of equations (5.1) with initial conditions (9.1) and $(a) N=1, L=0.005(\tilde{A}+\tilde{B}$ slowly oscillating, $\tilde{A}-\tilde{B}$ rapidly oscillating); $(b) N=1, L=20(\tilde{A}+\widetilde{B}$ rapidly oscillating, $\tilde{A}-\tilde{B}$ slowly oscillating). Abscissa $x$. (c) The detailed structure of $(a) ;(d)$ the detailed structure of $(b)$.

exist for all values of $L$ although they perhaps become more sparse as $L$ decreases. There are periodic solutions for non-zero cross-flow $N$, but not necessarily of predetermined period as in the case $N=0$.

When $L \gg 1$, so that $C$ and $A$ in (3.16) are completely in phase, all solutions of the difference equations of $\S 6$ appear to be periodic. Indeed, so do all solutions of (5.1) when the cross-flow is zero regardless of the starting conditions. Perhaps of most interest are flows with non-zero $N$ of which a little examination has been made here. At extreme values of $L$ (see $\S 9$ ) it is possible to compute solutions with $N \neq 0$ that have a fascinating periodic, or pseudo-periodic, structure; such asymptotic structures remain to be analysed. However at moderate values of $L, L=1$ for example, we have not been able to present solutions that are reproducible over more than a moderate distance in $x$. Any change in step length or accuracy tolerance has a devastating effect on the solution, which appears to become unbounded. This is in contrast with the situation when $L=1$ and $N=0$ where an arbitrary solution seems to remain bounded.

As noted above, the study in this paper is restricted to those values of the coefficients of the basic equations for which the solution neither terminates in a singularity nor decays exponentially. The autonomous system of equations considered is derived as an appropriate form of the integro-differential equations sufficiently far downstream that the effect of the initial conditions and of the non-parallelism of the basic flow at the point of input of the two waves is not of fundamental importance. Solutions for the amplitudes of the input waves that persisted over a large range of the streamwise variable were specifically sought. Although, in such a situation, the amplitudes are periodic or pseudo-periodic, their effect on the vortex solution, which satisfies the diffusion equation in the buffer region adjacent to the critical layer, is to promote its continual development over successive wave periods. This was also a feature of the single-wave input study of SBB. Such persistent interaction and consequent adjustment of the basic state is felt to be a possible pre-cursor of incipient transition and merits attention.

Finally, the present work is expected to lay the basis for further studies of increased non-symmetry, whether in the input amplitudes, in enlarged cross-flow, or in enlarged 
swirl (Part 2). It is desirable to deal with stronger cross-flows for theoretical and practical reasons and these may invoke full cross-flow waves, cf. the strong cross-flows considered by Davis \& Smith (1994).

The authors are grateful to $\operatorname{Dr} \mathrm{S}$. N. Timoshin for useful discussion and discerning comments.

\section{Appendix. The solutions of equation (6.5)}

When $L \gg 1$ a simple scaling reduces the homogeneous equation derived from (6.5) to the equation for simple harmonic motion and

in this limit.

$$
q \approx 1, \quad y_{0} \approx \pi L / 4+1, \quad r \approx 2^{-1 / 2} L, \quad y_{1}=O\left(L^{-2}\right)
$$

To discuss $\phi_{0}(x), \phi_{1}(x)$ when $L \ll 1$ it is convenient to define $y=\left(x-s_{n}\right) / \sqrt{ } 2$. The equation must be solved in two regions, namely $y=O(1)$ and $y=O\left(L^{1 / 2}\right)$. When $y=$ $O\left(L^{1 / 2}\right)$ we write $y=L^{1 / 2} z$, and find that, in the limit $L \rightarrow 0$, the solution $\tilde{C}_{0}$ of the homogeneous equation derived from (6.5) is

$$
\tilde{C}_{0}=z^{1 / 2}\left(\tilde{c}_{0} J_{-1 / 4}\left(z^{2}\right)+\tilde{d}_{0} J_{1 / 4}\left(z^{2}\right)\right),
$$

where $\tilde{c}_{0}, \tilde{d}_{0}$ are constants. When $y=O(1)$ a WKB solution is required and

$$
\tilde{C} \approx(\tanh y)^{-1 / 2}\left(\Gamma_{+} \mathrm{e}^{(2 \mathrm{i} / L) \log \cosh y}+\Gamma_{-} \mathrm{e}^{-(2 \mathrm{i} / L) \log \cosh y}\right)
$$

where $\Gamma_{+}, \Gamma_{-}$are also constants.

To obtain $q$ and $y_{0}$ we take

$$
\tilde{d}_{0}=0, \quad \tilde{c}_{0}=2^{-1 / 4}\left(-\frac{1}{4}\right) !
$$

in (A 2), and use the asymptotic form of the Bessel function as $z \rightarrow \infty$ to match with (A 3) as $y \rightarrow 0$. The result is that

$$
\Gamma_{ \pm}=\frac{\tilde{c}_{0}}{(2 \pi)^{1 / 2}} L^{1 / 4} \mathrm{e}^{\mp \mathrm{i} \pi / 8}
$$

and hence that, for $y \gg 1$,

$$
\tilde{C}_{0} \approx \tilde{c}_{0}(2 / \pi)^{1 / 2} L^{1 / 4} \sin \left[\frac{2 y}{L}-\frac{2}{L} \log 2+\frac{3 \pi}{8}\right] .
$$

This, together with (A 4), leads to

$$
q \approx \frac{(2 L)^{1 / 4}}{\pi^{1 / 2}}\left(-\frac{1}{4}\right) !, \quad y_{0} \approx \log 2-\frac{3 \pi}{16} L,
$$

when $L \ll 1$. On the other hand, to obtain $r$ and $y_{1}$ we take

$$
\tilde{c}_{0}=0, \quad \tilde{d}_{0}=2^{3 / 4}\left(\frac{1}{4}\right) ! L^{1 / 2}
$$

in (A 2) and find, instead of (A 5), that

$$
\Gamma_{ \pm}=\frac{\tilde{d}_{0}}{(2 \pi)^{1 / 2}} L^{1 / 4} \mathrm{e}^{\mp 3 i \pi / 8} .
$$




\begin{tabular}{rcccr}
\hline$L$ & $q($ num) & $q$ (a.e.) & $y_{0}$ (a.e.) & $y_{0}$ (num) \\
100 & 0.9999 & 1.000 & -77.5398 & -77.5403 \\
10 & 0.9925 & - & -6.8540 & -6.8600 \\
5 & 0.9726 & - & - & -2.9491 \\
4 & 0.9596 & - & - & -2.1742 \\
3 & 0.9360 & - & - & -1.4079 \\
2 & 0.8888 & - & - & -0.6598 \\
1 & 0.7841 & 0.8222 & 0.1040 & +0.0501 \\
0.8 & 0.7485 & 0.7775 & 0.2219 & 0.1852 \\
0.6 & 0.7031 & 0.7236 & 0.3397 & 0.3175 \\
0.4 & 0.6414 & 0.6538 & 0.4575 & 0.4466 \\
0.2 & 0.5445 & 0.5498 & 0.5753 & 0.5722 \\
0.1 & 0.4601 & 0.4623 & 0.6342 & 0.6333 \\
0.01 & 0.2599 & 0.2600 & 0.6872 & 0.6872
\end{tabular}

TABLE 3. Numerical values of $q$ and $y_{0}$ (columns 2 and 5) and the asymptotic values predicted by (A 1), (A 7) (columns 3 and 4).

$\begin{array}{rrccc}L & r \text { (num) } & r \text { (a.e.) } & y_{1} \text { (a.e.) } & y_{1} \text { (num) } \\ 100 & 70.7303 & 70.7107 & - & 0.0003 \\ 10 & 7.2586 & 7.0711 & - & 0.0309 \\ 5 & 3.8687 & - & - & 0.1049 \\ 4 & 3.2138 & - & - & 0.1475 \\ 3 & 2.5659 & - & - & 0.2162 \\ 2 & 1.9069 & - & - & 0.3273 \\ 1 & 1.1657 & 1.2163 & 0.4967 & 0.4924 \\ 0.8 & 0.9935 & 1.0288 & 0.5361 & 0.5310 \\ 0.6 & 0.8071 & 0.8292 & 0.5753 & 0.5709 \\ 0.4 & 0.6005 & 0.6118 & 0.6146 & 0.6116 \\ 0.2 & 0.3603 & 0.3838 & 0.6539 & 0.6527 \\ 0.1 & 0.2152 & 0.2163 & 0.6735 & 0.6731 \\ 0.01 & 0.0385 & 0.0385 & 0.6911 & 0.6911\end{array}$

TABLE 4. Numerical values of $r$ and $y_{1}$ (columns 2 and 5) and the asymptotic values produced by (A 1), (A 10) (columns 3 and 4).

Thus, for $y \gg 1$,

$$
\tilde{C}_{0} \approx \tilde{d}_{0}(2 / \pi)^{1 / 2} L^{1 / 4} \sin \left[\frac{2 y}{L}-\frac{2}{L} \log 2+\frac{\pi}{8}\right],
$$

this being the analogue of (A 6). Hence, finally, we obtain

$$
r \approx(2 L)^{3 / 4}(2 / \pi)^{1 / 2}\left(\frac{1}{4}\right) !, \quad y_{1} \approx \log 2-\frac{\pi}{16} L
$$

for $L \ll 1$.

In table 3 we present $q$ and $y_{0}$ found numerically for selected values of $L$ together with the results from the asymptotic formulae (A 7). Each $y_{0}$ is arbitrary to within a multiple of $L \pi$, so for definiteness each has been chosen so that $q>0$, and that, when $L=1,\left|y_{0}\right|$ is as small as possible, and that $y_{0}$ is a continuous function of $L$. Table 4 contains corresponding information for $r$ and $y_{1}$, the asymptotic formulae being (A 10). 


\section{REFERENCES}

Aihara, Y., Tomita, Y. \& Ito, A. 1984 Generation, development and distortion of longitudinal vortices in boundary layers along concave and flat plates. In Laminar-Turbulent Transition (ed. V. V. Kozlov), pp. 447-454. Springer.

Allen, T., Brown, S. N. \& SMIth, F. T. 1996 On vortex/wave interactions. Part 2. Originating from axisymmetric flow with swirl. (Submitted.)

Bassom, A. P. \& GAJJAR, J. S. B. 1988 Non-stationary cross-flow vortices in three-dimensional boundary-layer flows. Proc. R. Soc. Lond. A 417, 179-212.

BenNEY, D. J. \& ChOw, C. 1989 A mean flow first harmonic theory for hydrodynamic instabilities. Stud. Appl. Maths 80, 37.

BlenNERHASSETT, P. \& SMITH, F. T. 1992 Nonlinear interactions of oblique three-dimensional Tollmien-Schlichting waves and longitudinal waves, in channel flows and boundary layers. Proc. $R$. Soc. Lond. A 436, 585-602.

Brown, P. G., Brown, S. N., Smith, F. T. \& Timoshin, S. N. 1993 On the starting process of strongly nonlinear vortex/Rayleigh-wave interactions. Mathematika 40, 7-29 (BBST).

Davis, D. A. R. \& Smith, F. T. 1994 Influence of cross-flow on nonlinear TollmienSchlichting/vortex interaction. Proc. R. Soc. Lond. A 446, 319-340.

GAJJAR, J.S. B. 1995 On the nonlinear instability of stationary cross-flow vortices in threedimensional boundary layers. (In preparation.)

GoldsteIN, M. E. \& CHOI, S.-W. 1989 Nonlinear evolution of interacting oblique waves on twodimensional shear layers. J. Fluid Mech. 207, 97-120 (and corrigendum, 216, 1990, 659-663).

HALL, P. 1986 An asymptotic investigation of the stationary modes of instability of the boundary layer on a rotating disc. Proc. $R$. Soc. Lond. A 406, 93-106.

HaLl, P. \& SMITH, F. T. 1988 The nonlinear interaction of Tollmien-Schlichting waves and Taylor-Gortler vortices in curved channel flows. Proc. R. Soc. Lond. A 417, 255-282.

HaLl, P. \& SMITH, F. T. 1989 Nonlinear Tollmien-Schlichting/vortex interaction in boundary layers. Eur. J. Mech. B 8, 179-205.

HaLL, P. \& SмITH, F. T. 1991 On strongly nonlinear vortex/wave interactions in boundary-layer transition. J. Fluid Mech. 227, 641-666.

Hall, P. \& SMITH, F. T. 1990 Near-planar TS waves and longitudinal vortices in channel flow: nonlinear interaction and focusing. ICASE Rep. 89-47.

KHoKhLOv, A. P. 1994 The theory of resonance interaction of Tollmien-Schlichting waves. J. Appl. Mech. Tech. Phys. 34, 508-515.

Klebanoff, P. S. \& Timstrom, K. D. 1959 Evolution of amplified waves leading to transition in a boundary layer with zero pressure gradient. NASA Tech. Note D-195.

KLEISER, L. \& ZANG, T. A. 1991 Numerical simulation of transition in wall-bounded shear flows. Ann. Rev. Fluid Mech. 23, 495-537.

Kohama, Y., Saric, W. S. \& Noos, J. A. 1991 A high frequency secondary instability of crossflow vortices that leads to boundary-layer transition. In Boundary Layer Transition and Control. $R$. Aero. Soc. Symp. Cambridge University Press.

NishIOKA, M., ASAI, N. \& IIDA, S. 1979 An experimental investigation of the secondary instability. In Laminar-Turbulent Transition, IUTAM Symp. Stuttgart. Springer.

REED, H. \& SARIC, W. S. 1989 Stability of three-dimensional boundary layers. Ann. Rev. Fluid Mech. $21,235-284$.

REMPFER, D. \& FASEL, H. F. 1994 Evolution of three-dimensional coherent structures in a flat-plate boundary layer. J. Fluid Mech. 260, 351-375.

SAndham, N. D. \& Kleiser, L. 1992 The late stages of transition to turbulence in channel flow. $J$. Fluid Mech. 245, 319-348.

SMITH, F. T. \& Bowles, R. I. 1992 Transition theory and experimental comparisons on (I) amplification into streets and (II) a strongly nonlinear break-up criterion. Proc. R. Soc. Lond. A 439, 163-175.

Smith, F. T., Brown, S. N. \& Brown, P. G. 1993 Initiation of three-dimensional nonlinear transition paths from an inflexional profile. Eur. J. Mech. B12, 447-473. (SBB.)

SPALART, P. R. \& YANG, K.-S. 1987 Numerical study of ribbon-induced transition in Blasius flow. J. Fluid Mech. 178, 345-365. 
Stewart, P. A. \& Smith, F. T. 1987 Three-dimensional instabilities in steady and unsteady nonparallel boundary layers, including effects of Tollmien-Schlichting disturbances and crossflow. Proc. R. Soc. Lond. A 409, 229-248.

Stewart, P. A. \& SMith, F. T. 1992 Three-dimensional nonlinear blow-up from a nearly planar initial disturbance in boundary-layer transition: theory and experimental comparisons. J. Fluid Mech. 244, 79-100.

StUaRT, J. T. 1963 Hydrodynamic Stability. In Laminar Boundary Layers (ed. L. Rosenhead), Chap. IX. Oxford University Press.

Tani, I. \& Sakagami, J. 1962 In Proc. Intl Council on Aeron. Sci., pp. 391-403, Third Congress, Stockholm. New York: Spartan.

Timoshin, S. N. \& SмiтH, F. T. 1995 Vortex/inflectional-wave interactions with weakly threedimensional input. (Submitted.)

Walton, A. G., Bowles, R. I. \& Smith, F. T. 1994 Vortex-wave interaction in separating flows. Eur. J. Mech. B 13, 629-655.

Walton, A. G. \& SMith, F. T. 1992 Properties of strong nonlinear vortex/Tollmien-Schlichting interactions. J. Fluid Mech. 244, 649-676.

Wray, A. \& Hussaini, M. Y. 1984 Numerical experiments in boundary-layer stability. Proc. R. Soc. Lond. A 392, 373-389.

Wu, X. 1993 a Nonlinear temporal-spatial modulation of near-planar Rayleigh waves in shear flows: formation of streamwise vortices. J. Fluid Mech. 256, 685-719.

Wu, X. $1993 b$ On critical-layer and diffusion-layer nonlinearity in the three-dimensional stage of boundary-layer transition. Proc. R. Soc. Lond. A 433, 95-106.

Wu, X. 1995 Viscous effects on fully coupled resonant-triad interactions: an analytical approach. J. Fluid Mech. 292, 377-407.

Wu, X. \& COWLEY, S. J. 1995 On the nonlinear evolution of instability modes in unsteady shear layers: the Stokes layer as a paradigm. Q. J. Mech. Appl. Maths 48, 159-188.

Wu, X., LeE, S. S. \& CowleY, S. J. 1993 On the weakly nonlinear three-dimensional instability of shear layers to pairs of oblique waves: the Stokes layer as a paradigm. J. Fluid Mech. 253, $681-721$. 